TRANSACTIONS OF THE

AMERICAN MATHEMATICAL SOCIETY

Volume 351, Number 3, March 1999, Pages 1171-1201

S 0002-9947(99)02346-6

\title{
BUILDING BLOCKS FOR QUADRATIC JULIA SETS
}

\author{
JOACHIM GRISPOLAKIS, JOHN C. MAYER, AND LEX G. OVERSTEEGEN
}

\begin{abstract}
We obtain results on the structure of the Julia set of a quadratic polynomial $P$ with an irrationally indifferent fixed point $z_{0}$ in the iterative dynamics of $P$. In the Cremer point case, under the assumption that the Julia set is a decomposable continuum, we obtain a building block structure theorem for the corresponding Julia set $J=J(P)$ : there exists a nowhere dense subcontinuum $B \subset J$ such that $P(B)=B, B$ is the union of the impressions of a minimally invariant Cantor set $A$ of external rays, $B$ contains the critical point, and $B$ contains both the Cremer point $z_{0}$ and its preimage. In the Siegel disk case, under the assumption that no impression of an external ray contains the boundary of the Siegel disk, we obtain a similar result. In this case $B$ contains the boundary of the Siegel disk, properly if the critical point is not in the boundary, and $B$ contains no periodic points.

In both cases, the Julia set $J$ is the closure of a skeleton $S$ which is the increasing union of countably many copies of the building block $B$ joined along preimages of copies of a critical continuum $C$ containing the critical point. In addition, we prove that if $P$ is any polynomial of degree $d \geq 2$ with a Siegel disk which contains no critical point on its boundary, then the Julia set $J(P)$ is not locally connected. We also observe that all quadratic polynomials which have an irrationally indifferent fixed point and a locally connected Julia set have homeomorphic Julia sets.
\end{abstract}

\section{INTRODUCTION}

1.1. Definitions and notation. We denote the complex plane by $\mathbb{C}$, the Riemann sphere by $\mathbb{C}_{\infty}$, and the open unit disk by $\mathbb{D}$. For a set $A$, we denote the closure of $A$ by $\bar{A}$ and the boundary of $A$ by $\partial A$, it being understood which of the above spaces $A$ is a subset of. We identify $\partial \mathbb{D}$ with $\mathbb{T} \equiv \mathbb{R} / \mathbb{Z}$ so that we may measure angles in revolutions. In what follows, arithmetic operations on $t \in \mathbb{T}$, for example $2^{n} t, t+\alpha$, etc., are to be taken modulo 1 unless context forbids. For $a, b, c \in \mathbb{T}$, we will denote by $[a, b, c]$ the interval in $\mathbb{T}$ with endpoints $a$ and $c$ containing the point $b$.

Let $P$ be a polynomial (or more generally, a rational function or entire function). The Julia set of $P$, denoted $J(P)$, is defined to be the set of all points in $\mathbb{C}\left(\mathbb{C}_{\infty}\right.$ for rational functions) at which $\left\{P^{n}\right\}_{n=0}^{\infty}$ is not normal. The complement of the

Received by the editors August 30, 1995.

1991 Mathematics Subject Classification. Primary 30C35, 54F20.

Key words and phrases. Julia set, complex analytic dynamics, prime ends, decomposable continuum, quadratic polynomial, Siegel disk, Cremer point, irrationally indifferent fixed point, rotation number.

Portions of this paper were presented at the Spring Topology Conference in Auburn, Alabama, March 1994, and in the special session on Geometry of Dynamical Systems at the AMS meeting in Orlando, Florida, March 1995. 
Julia set is the Fatou set, or stable set, denoted $F(P)$. The orbit of a point $z$ under $P$ is $\mathcal{O}(z)=\mathcal{O}_{P}(z)=\left\{P^{n}(z)\right\}_{n=0}^{\infty}$. We shall sometimes have occasion to refer to the inverse orbit $\mathcal{O}^{-}(z)=\left\{y \mid \exists n \in \mathbb{N}, P^{n}(y)=z\right\}$, and the grand orbit $\mathcal{G}(z)=\left\{y \mid \exists n, m \in \mathbb{N}, P^{n}(y)=P^{m}(z)\right\}$. A point $z$ is preperiodic iff its orbit is finite, is periodic iff for some $n \in \mathbb{Z}^{+}, P^{n}(z)=z$, and is fixed iff $n=1$. For $z$ a periodic point of least period $n$, we call $\lambda(z)=\left(P^{n}\right)^{\prime}(z)$ the eigenvalue of the periodic orbit. A periodic point is attracting, indifferent, or repelling accordingly as $|\lambda|<1,|\lambda|=1$, or $|\lambda|>1$, respectively. A subset $A \subset \mathbb{C}_{\infty}$ is invariant iff $P(A) \subset A$ and fully invariant iff $P^{-1}(A)=A$.

In this paper we consider only the case where $P$ is a polynomial. By theorems of Julia and Fatou, generalized by Baker, the Julia set is the closure of the set of repelling periodic points of $P$. Attracting periodic points are always in the Fatou set. Indifferent periodic points may be in either the Julia set or the Fatou set, and this dichotomy forms a focus of our paper. Basic theorems concerning Julia and Fatou sets may be found in any of several introductions to complex dynamics, for instance those by Milnor [20], Beardon [2], or Carleson and Gamelin [8]. Important facts we shall use are Sullivan's No Wandering Domains Theorem (all bounded Fatou components are, as sets, preperiodic $[33,34]$ ), and the fact that the Julia set of a polynomial of degree $d \geq 2$ is compact, perfect, nonempty, and bounded away from $\infty[20,8]$. In addition, an attractive or indifferent orbit always attracts (in an appropriate sense) a critical point [8]. A continuum is a compact connected metric space. The Julia set is connected, hence a continuum, iff the orbit of every critical point is bounded, as we discuss in Section 1.2.

Our attention in this paper will be focused on quadratic polynomials such that one of the two fixed points is indifferent. Unless there is only one fixed point, the other fixed point is then repelling. Let $z_{0}$ denote an indifferent fixed point. Then $\lambda\left(z_{0}\right)=e^{2 \pi i \alpha}$ for some $\alpha\left(z_{0}\right) \in \mathbb{T}$. If $\alpha=\alpha\left(z_{0}\right)$ is rational, then $z_{0}$ is in the Julia set, the so-called parabolic case. In this case the Julia set is a locally connected continuum whose structure is well-understood [20]. If $\alpha=\alpha\left(z_{0}\right)$ is irrational, there are two possibilities:

1. $z_{0}$ is a Cremer point if $z_{0}$ is in the Julia set.

2. $z_{0}$ is a Siegel point if $z_{0}$ is in the Fatou set.

Which branch of the dichotomy $z_{0}$ falls into depends upon how irrational $\alpha\left(z_{0}\right)$ is.

Consider the irrational number $\alpha$, and let $\left\langle a_{0}, a_{1}, \ldots, a_{n}, \ldots\right\rangle$ be the continued fraction expansion of $\alpha$ and $p_{n} / q_{n}$ the convergents defined by the recurrences $p_{0}=$ $q_{1}=1$ and $p_{1}=q_{0}=0, p_{n+1}=a_{n+1} p_{n}+p_{n-1}$ and $q_{n+1}=a_{n+1} q_{n}+q_{n-1}$. Assume, also, that $a_{0}=0$, so $0<\alpha<1$. We say that $\alpha$ is a $k$-Diophantine number, for $k>0$, provided that there exists a number $c>0$ such that for all $n$

$$
\left|\alpha-\frac{p_{n}}{q_{n}}\right| \geq \frac{c}{q_{n}^{k}} .
$$

The union of all the sets of $k$-Diophantine numbers is called the class of Diophantine numbers. The remaining irrational numbers are called Liouville numbers and form a dense $G_{\delta}$ subset of $\mathbb{R}$ with Lebesgue measure 0 . We say that the number $\alpha$ is of constant type provided that $\sup a_{n}<\infty$. An irrational number is called a Brjuno number provided that

$$
\sum \frac{\log q_{n+1}}{q_{n}}<\infty
$$


All Diophantine numbers are Brjuno numbers, though not conversely.

An irrationally indifferent fixed point $z_{0}$ of a polynomial is in the Fatou set iff it is locally linearizable at $z_{0}$ : there is a complex analytic homeomorphism $\phi: \mathbb{D} \rightarrow U_{0}$, $U_{0}$ a neighborhood of $z_{0}$, such that for all $z \in \mathbb{D}, P(\phi(z))=\phi\left(r_{\alpha}(z)\right)$, where $r_{\alpha}(z)=e^{2 \pi i \alpha} z$ is a rigid rotation of the unit disk through angle $\alpha \in \mathbb{T}$. That is, $\phi$ conjugates $P$ on $U_{0}$ to $r_{\alpha}$ on $\mathbb{D}$. In this case, the fixed point $z_{0}$ belongs to an invariant bounded Fatou component $\Delta$, which is maximal with respect to conjugating $P$ to the linear map $r_{\alpha}$, and which is called a Siegel disk. Otherwise, $z_{0}$ belongs to the Julia set $J(P)$ and is called a Cremer point. Siegel [30] showed that Siegel disks occur and Cremer [9] showed that Cremer points occur. By results of Brjuno [3] and J.C. Yoccoz [36, 35] a quadratic polynomial is locally linearizable at the irrationally indifferent fixed point $z_{0}$ if and only if the argument $\alpha$ is a Brjuno number. For a quadratic polynomial, the presence of an indifferent fixed point, parabolic, Cremer, or Siegel, forces the critical point to have a bounded orbit [8], which in turn forces the Julia set to be a continuum as we discuss below.

1.2. External rays. For $P$ a polynomial map of $\mathbb{C}$ of degree $d \geq 2$, let $K(P)$ denote the "filled-in" Julia set: $J(P)$ together with all its bounded complementary domains, if any. Equivalently, $K(P)$ is the set of points with bounded orbit. Let $U_{\infty}=\mathbb{C}_{\infty} \backslash K(P)$. This notation is appropriate since the domain $\mathbb{C}_{\infty} \backslash K(P)$ is the basin of attraction of $\infty$; under iteration of $P$ all points in $U_{\infty}$ tend to $\infty$. It can be proved that $\partial K(P)=\partial U_{\infty}=J(P)$ [20, Problem 4.1 and Lemma 17.1].

Prime end theory, introduced by Carathéodory, is a way of studying the approaches to the boundary of a simply connected domain $U$ with nondegenerate boundary. The Riemann mapping theorem guarantees the existence of a conformal isomorphism $\phi$ from the unit disk $\mathbb{D}$ onto $U$. Prime end theory is one of the most important tools for studying the topological structure of the boundaries of Fatou components, and consequently, of Julia sets.

It is a consequence of a classical theorem of Bötkher [20, Theorem 6.7] that if $P$ is a polynomial map of $\mathbb{C}$ of degree $d \geq 2$, then there is a neighborhood $N_{\infty}$ of infinity on which $P$ is analytically conjugate to the map $z \rightarrow z^{d}$ near $\infty$. To be more precise, let $D_{r}^{\infty}=\mathbb{C}_{\infty} \backslash \overline{\mathbb{D}}_{r}$, for some $r>1$, be a disk about $\infty$. Then there exists a conformal isomorphism $\psi: N_{\infty} \rightarrow D_{r}^{\infty}$ taking $\infty$ to $\infty$ such that $\psi(P(z))=\psi(z)^{d}$. In general, $\psi$ cannot be extended as a conformal isomorphism from the complement $U_{\infty}$ of the filled-in Julia set $K(P)$ (to some disk), because the critical points of $P$ in $U_{\infty}$ are an obstruction to the extension. However, if all the critical points are in $K(P)$, then there is no obstruction, and $\psi$ can be extended to a conformal isomorphism $\psi: U_{\infty} \rightarrow \mathbb{C}_{\infty} \backslash \overline{\mathbb{D}}$, unique up to multiplication by a $(d-1)$ st root of unity [20, Theorem 17.3$]$.

Let $\mathbb{D}_{\infty}=\mathbb{C}_{\infty} \backslash \overline{\mathbb{D}}$ denote the "unit disk" about $\infty$. Suppose each critical point of $P$ has a bounded orbit. It follows from the above remarks that there is a canonical conformal map $\phi: \mathbb{D}_{\infty} \rightarrow U_{\infty}$ (namely $\psi^{-1}$ from above) taking $\infty$ to $\infty$. This map is called the Bötkher uniformization of $U_{\infty}=\mathbb{C}_{\infty} \backslash K(P)$. The map $\phi^{-1} P \phi$ on $\mathbb{D}_{\infty}$ is $z \rightarrow z^{d}$. The induced map on $\mathbb{T}=\partial \mathbb{D}=\partial \mathbb{D}_{\infty}$ is $\sigma_{d}: t \rightarrow d t(\bmod 1)$. Moreover, since $K(P)$ is the intersection of the disks $K_{r}=\mathbb{C}_{\infty} \backslash \phi\left(\mathbb{D}_{r}^{\infty}\right)$, for $r>1$, the filled-in Julia set is connected, as is its boundary $J(P)$.

The radial rays $\left\{r e^{2 \pi i t} \mid r>1\right\}$ from $\infty \in \mathbb{D}_{\infty}$ are carried by $\phi$ to rays $R_{t}=$ $\phi\left(\left\{r e^{2 \pi i t} \mid r>1\right\}\right)$ from $\infty \in U_{\infty}$ which accumulate on $\partial U_{\infty}=J(P)$. Such rays are called external rays in complex dynamics literature. Each point $t \in \mathbb{T}$, via the ray 
$R_{t}$, determines a unique prime end of $U_{\infty}$. The limit set $\operatorname{Pr}\left(R_{t}\right)=\bar{R}_{t} \backslash R_{t} \subset J(P)$ is the principal continuum of the prime end corresponding to $t \in \mathbb{T}$. Since it is the limit set of a ray, it is easy to see that the principal continuum corresponding to $t$ is indeed a continuum.

Associated with each external ray is another continuum in $J(P)$ which always contains the principal continuum, but may be larger. The impression of $R_{t}$, denoted $\operatorname{Im}\left(R_{t}\right)$, is defined as

$$
\operatorname{Im}\left(R_{t}\right)=\left\{z \in \mathbb{C} \mid \exists w_{i} \in \mathbb{D}_{\infty}, w_{i} \rightarrow t, \phi\left(w_{i}\right) \rightarrow z\right\} .
$$

The following result follows easily from the definition:

Lemma 1.1. If $t_{i} \rightarrow t_{0}$, then $\lim \sup \operatorname{Im}\left(R_{t_{i}}\right) \subset \operatorname{Im}\left(R_{t_{0}}\right)$.

Applying the Bötkher uniformization and the above definitions, one can prove the following lemma showing that $P$ permutes principal continua and impressions as $\sigma_{d}$ permutes angles.

Lemma 1.2 ([19, Lemma 2.1]). Let $P$ be a polynomial of degree $d \geq 2$ with all critical orbits bounded and $\phi$ the canonical Bötkher uniformization of $\mathbb{C}_{\infty} \backslash K(P)$. Then for all $t \in \mathbb{T}, P\left(\operatorname{Im}\left(R_{t}\right)\right)=\operatorname{Im}\left(R_{d t}\right)$ and $P\left(\operatorname{Pr}\left(R_{t}\right)\right)=\operatorname{Pr}\left(R_{d t}\right)$.

Note that for an external ray $R_{t}, \bar{R}_{t}=R_{t} \cup \operatorname{Pr}\left(R_{t}\right)$. By $\widehat{R}_{t}$ we denote the set $R_{t} \cup \operatorname{Im}\left(R_{t}\right)$, sort of a "superclosure" of the external ray. The external rays "foliate" $U_{\infty}$ in the sense that each point of $U_{\infty}$, except $\infty$, is in exactly one external ray. The polynomial $P$ then preserves this foliation, and extends this preservation to principal continua and impressions within the boundary of $U_{\infty}$. However, closing the leaves of the foliation does not generally produce a foliation of $\bar{U}_{\infty}$, since $\bar{R}_{t}$ may meet $\bar{R}_{s}$ even if $t \neq s$, and $\bigcup_{t \in \mathbb{T}} \operatorname{Pr}\left(R_{t}\right)$ may be properly contained in $\partial U_{\infty}$. On the other hand, though $\operatorname{Im}\left(R_{t}\right)$ may meet $\operatorname{Im}\left(R_{s}\right)$ even if $t \neq s$, it is the case that $\bigcup_{t \in \mathbb{T}} \operatorname{Im}\left(R_{t}\right)=\partial U_{\infty}$.

The following is a classical theorem of Carathéodory:

Theorem 1.3 (Carathéodory Extension Theorem). The uniformization $\phi: \mathbb{D}_{\infty} \rightarrow$ $U_{\infty}$ extends continuously to $\overline{\mathbb{D}}_{\infty}$ if, and only if, $\partial U_{\infty}$ is locally connected.

Hence, when such an extension exists, the Julia set $J(P)=\partial U_{\infty}$ is both connected and locally connected. The extension of $\phi$ is defined in the obvious way: $\phi$ extends continuously to carry $\mathbb{T}=\partial \mathbb{D}$ onto $\partial U_{\infty}$ by mapping $t \in \mathbb{T}$ to $\operatorname{Pr}\left(R_{t}\right)=z_{t} \in \partial U_{\infty}$, which is a single point in this case.

It is easy to see that if $J(P)$ is locally connected, then all external rays "land"; that is, all principal continua are degenerate. It turns out this condition is not sufficient for a Julia set to be locally connected [10]. However, some rays always land, since accessible points of $\partial U_{\infty}$ are dense in $\partial U_{\infty}$. In the case of a polynomial with a connected Julia set, we have the following additional information:

Theorem 1.4 (Douady/Hubbard $[20,18.1,18.4]$ ). If $R_{t}$ is a radial ray with rational argument $t \in \mathbb{T}$, the principal set of $R_{t}$ is a single point, which is either periodic, and repelling or indifferent with rational argument, or preperiodic to such a periodic point.

Theorem 1.5 (Douady/Yoccoz $[20,18.2,18.3])$. If $z$ is a repelling or rationally indifferent periodic point in $J(P)$, then at least one external ray lands on $z$, necessarily periodic, and at most finitely many do, all of the same period. 
1.3. Locally connected Julia sets. Douady and Hubbard (independently: Sullivan, Lyubich) found sufficient conditions for the Julia set of a polynomial to be locally connected.

Theorem 1.6 (Douady/Hubbard [20, 17.5]). If all critical points of a polynomial are either attracted to an attracting periodic orbit, or preperiodic, then the Julia set is locally connected.

They show that the Bötkher uniformization extends continuously, and apply the Carathéodory Extension Theorem 1.3. In the case of a quadratic polynomial $P$, there is only one critical point, and each of the following mutually exclusive conditions is sufficient for the Julia set to be a locally connected continuum [20]:

1. $P$ has an attractive periodic orbit.

2. $P$ has a rationally indifferent (parabolic) periodic orbit.

3. $P$ has a preperiodic critical orbit.

More recently, using new methods, Yoccoz has proved the following theorem [21]:

Theorem 1.7 (Yoccoz). Let $P$ be a quadratic polynomial with a connected Julia set $J=J(P)$. If $P$ has no irrationally indifferent periodic points, and $P$ is not infinitely renormalizable, then $J$ is locally connected.

1.4. Connected, but not locally connected, Julia sets. If a polynomial (with a connected Julia set, if you like) has an irrationally indifferent periodic orbit, the question of the Julia set's local connectivity leads to some subtleties. One accessible result is the following:

Theorem 1.8 (Douady, Sullivan [20, 18.6]). If $P$ is a polynomial with a Cremer orbit, then $J(P)$ is not locally connected.

By a similar proof, a general version of which we shall present in Section 2.1, one can obtain the following:

Theorem 1.9 (Douady). If $P$ is a quadratic polynomial with an invariant Siegel disk $\Delta$, and the critical point is not in $\partial \Delta$, then $J(P)$ is not locally connected.

The proofs of the above theorems offer little insight into the topological structure of the Julia sets. We report on what is known about the structure of non-locallyconnected Julia sets with irrationally indifferent orbits in Section 3, and state our own observations in the main theorems (Section 4) of this paper. We do not address infinitely renormalizable polynomials.

\section{TWO EASY PIECES}

We present the fairly simple proofs of two theorems which serve to introduce some of the concepts we will call upon later in less transparent situations. First we observe the following two facts which we will frequently use:

Theorem 2.1 (Rogers [26]). Let $\Delta$ be a Siegel disk for a polynomial P. Then $\partial \Delta$ is an irreducible separator of the plane.

Proof. The result follows from the fact that $\partial \Delta \subset \bar{\Delta} \cap \overline{U_{\infty}}$.

Theorem 2.2. Let $J$ be a locally connected Julia set of a polynomial P. Then every subcontinuum of $J$ is locally connected. 
Proof. Let $G$ be a subcontinuum of the locally connected Julia set $J$ of a polynomial $P$. Suppose that $G$ is not locally connected. Then there exists a nondegenerate continuum of convergence $K$ in $G$ [18, p. 245]. Hence, there exist pairwise disjoint continua $K_{i}$ in $G$ such that $\lim K_{i}=K$ and $K_{i} \cap K=\emptyset$ for each $i$. Choose $x \neq y \in K$ and closure-disjoint connected neighborhoods $U$ and $V$ of $x$ and $y$, respectively, since $J$ is locally connected. Then there exist $i_{1}, i_{2}, i_{3}$ such that $K_{i_{2}}$ meets a bounded complementary domain of $K_{i_{1}} \cup \bar{U} \cup \bar{V} \cup K_{i_{3}}$. This contradicts the fact that $J \subset \overline{U_{\infty}}$.

2.1. Siegel disks with no critical points in their boundary. Theorem 1.9 is usually quoted in the literature exclusively with reference to quadratic polynomials. However, its proof for all polynomials of degree $d \geq 2$ with connected Julia set is straightforward. We present it below to call attention to the interplay between $P$ on external rays and their principal sets (and impressions, later on) and the map $\sigma$ induced on $\mathbb{T}$ by the Bötkher uniformization. Let $(X, d)$ be a metric space. We will call a map $f: X \rightarrow X$ forward expansive provided there exist $\epsilon>0$ and $k>1$ such that for all $x, y \in X$, if $d(x, y)<\epsilon$, then $d(f(x), f(y)) \geq k d(x, y)$.

Theorem 2.3 ([20, 18.8]). Let $h: X \rightarrow X$ be a forward expansive homeomorphism. If $X$ is compact, then $X$ is finite.

Corollary 2.4. Let $\sigma_{d}: \mathbb{T} \rightarrow \mathbb{T}(d \geq 2)$ be given by $\sigma_{d}(t)=d t$. Let $A$ be a compact subset of $\mathbb{T}$. If $\sigma(A)=A$ and $\left.\sigma\right|_{A}$ is one-to-one, then $A$ is finite.

Lemma 2.5. Let $P$ be a polynomial of degree $d \geq 2$ and $H$ an invariant compact subset of $\mathbb{C}$ such that $P$ is univalent on $H$. If no critical point lies in $\partial H$, then $P$ is univalent on a neighborhood of $H$.

Proof. Let $\left\{U_{i}\right\}_{i=1}^{\infty}$ be a sequence of $\frac{1}{i}$-neighborhoods of $H$. By way of contradiction, suppose for all $i, P$ is not univalent on $U_{i}$. Then there are sequences $\left\{z_{i}\right\}$, $\left\{z_{i}^{\prime}\right\}$ with $z_{i}, z_{i}^{\prime} \in U_{i}$ such that $P\left(z_{i}\right)=P\left(z_{i}^{\prime}\right)$. By choosing subsequences, we may assume $z_{i} \rightarrow z_{0}$ and $z_{i}^{\prime} \rightarrow z_{0}^{\prime}$. Then $z_{0}, z_{0}^{\prime} \in \partial H$. If $z_{0}=z_{0}^{\prime}$, then $z_{0}$ is a critical point in $\partial H$, a contradiction. If $z_{0} \neq z_{0}^{\prime}$, then as $P\left(z_{0}\right)=P\left(z_{0}^{\prime}\right)$ we contradict the univalence of $P$ on $H$.

Theorem 2.6. Let $P$ be a polynomial of degree $d \geq 2$ with connected Julia set $J=J(P)$ and $\Delta$ an invariant Siegel disk of $P$. If no critical point lies in $\partial \Delta$, then $J$ is not locally connected.

Proof. By way of contradiction, suppose $J$ is locally connected. By Theorems 2.1 and 2.2, $\partial \Delta$ is also locally connected and an irreducible separator of $\mathbb{C}$ and, hence, $\partial \Delta$ is homeomorphic to a circle. Then the Riemann map conjugating $\left.P\right|_{\Delta}$ to an irrational rotation on $\mathbb{D}$ extends homeomorphically to $\partial \Delta$ and $\left.P\right|_{\bar{\Delta}}$ is conjugate to an irrational rotation of $\overline{\mathbb{D}}$. Hence, $P$ is univalent on $\bar{\Delta}$. By Lemma 2.5, $P$ is univalent on a neighborhood of $\bar{\Delta}$. Let $\phi: \mathbb{C}_{\infty} \backslash \overline{\mathbb{D}} \rightarrow \mathbb{C}_{\infty} \backslash K(P)$ be the Bötkher uniformization. Since $K=K(P)$ is locally connected, $\phi$ extends continuously to carry $\mathbb{T}=\partial \mathbb{D}$ onto $J=\partial K$ by mapping $t \in \mathbb{T}$ to $\operatorname{Pr}\left(R_{t}\right)=z_{t} \in J$, a single point. Define

$$
A=\left\{t \in \mathbb{T} \mid \operatorname{Pr}\left(R_{t}\right)=z_{t} \in \partial \Delta\right\} .
$$

Since $\partial \Delta$ has infinitely many points, $A$ is infinite. Since $\phi$ extends continuously, $A$ is the preimage of the compact set $\partial \Delta$, so is compact. Since $P$ is univalent on a neighborhood of $\partial \Delta$ and $\partial \Delta$ is invariant, the external rays which land on 
$\partial \Delta$ must be permuted one-to-one by $P$. Thus, the map $\sigma_{d}$ induced on $\mathbb{T}$ by the uniformization must be one-to-one on $A$. By Corollary 2.4, $A$ must be finite, a contradiction.

2.2. Locally connected quadratic Julia sets with a Siegel disk. Our goal in this section is to prove that all locally connected quadratic Julia sets with an invariant Siegel disk in the complementary Fatou set are homeomorphic, regardless of the differing dynamics.

Theorem 2.7. Let $P_{1}$ and $P_{2}$ be quadratic polynomials, each with a linearizable fixed point and with locally connected Julia set. Then $J\left(P_{1}\right)$ and $J\left(P_{2}\right)$ are topologically equivalent.

To prove Theorem 2.7, we shall first prove a "Building Block" theorem for these polynomials. In Section 6 we prove the general Building Block Theorem 4.1 in the Siegel disk case, without the assumption that the Julia set is locally connected. That Theorem 2.7 is nonempty is a consequence of a striking result by C. L. Petersen $[25]$.

Theorem 2.8 (Petersen). If $P(z)=e^{2 \pi i \alpha} z+z^{2}$, where $\alpha$ is an irrational Diophantine number of constant type, then the Julia set $J(P)$ is locally connected.

Corollary 2.9. For all quadratic polynomials of the form $P_{\alpha}(z)=e^{2 \pi i \alpha} z+z^{2}$, where $\alpha$ is an irrational Diophantine number of constant type, the resulting Julia sets $J\left(P_{\alpha}\right)$ are homeomorphic.

Proof. It is a classical theorem of Siegel [30] that $P$ is linearizable at 0 . The corollary is a direct consequence of Theorems 2.7 and 2.8 .

Theorem 2.10. Let $P(z)=e^{2 \pi i \alpha} z+z^{2}$ be a polynomial with an invariant Siegel disk $\Delta$ such that $J=J(P)$ is locally connected. Then $\partial \Delta$ is a Jordan curve denoted by $B$, and there is a unique Cantor set $A \subset \mathbb{T}$ such that the following hold:

1. $B$ is carried homeomorphically onto itself by $P$.

2. The critical point $c$ of $P$ belongs to $B$, and $B=\overline{\mathcal{O}_{P}(c)}$.

3. There is a unique $\theta \in \mathbb{T}$ such that $R_{\theta}$ lands on $v=P(c)$.

4. $A=\overline{\mathcal{O}_{\sigma}(\theta)}$.

5. $A \subset\left[\frac{\theta}{2}, \theta, \frac{\theta+1}{2}\right]$, where $\left[\frac{\theta}{2}, \theta, \frac{\theta+1}{2}\right]$ is the half circle in $\mathbb{T}$ from $\frac{\theta}{2}$ to $\frac{\theta+1}{2}$ containing $\theta$.

6. $\sigma(A)=A$, minimally, with rotation number $\alpha$.

7. Let $\phi: \overline{\mathbb{D}}_{\infty} \rightarrow \bar{U}_{\infty}$ be the extension of the Bötkher uniformization. Then $\phi(A)=B$, one-to-one except onto $\mathcal{O}_{P}^{-}(c) \cap B$.

8. The diameters of components of $P^{-n}(B)$ go to 0 as $n \rightarrow \infty$.

9. $J \backslash \bigcup_{n=0}^{\infty} P^{-n}(B)$ is a fully invariant, 0-dimensional, dense $G_{\delta}$ set in $J$ containing all the (pre)periodic points of $J$.

Proof. As in the proof of Theorem 2.6, $B=\partial \Delta$ is a Jordan curve, $P(B)=B$, and $\left.P\right|_{B}$ is conjugate to the irrational rotation $r_{\alpha}$. Since $J$ is locally connected, Theorem 2.6 implies that the critical point $c$ lies on $B$. Conjugacy to an irrational rotation implies $P$ is one-to-one and $\mathcal{O}(c)$ is dense in $B$, which establishes conclusions (1) and (2).

Since $J(P)$ is locally connected, the Carathéodory Extension Theorem 1.3 implies that every point of $J(P)$ is the landing point of some external ray. Let $R_{\theta}$ be an external ray landing at the critical value $v=P(c)$ which proves the existence 


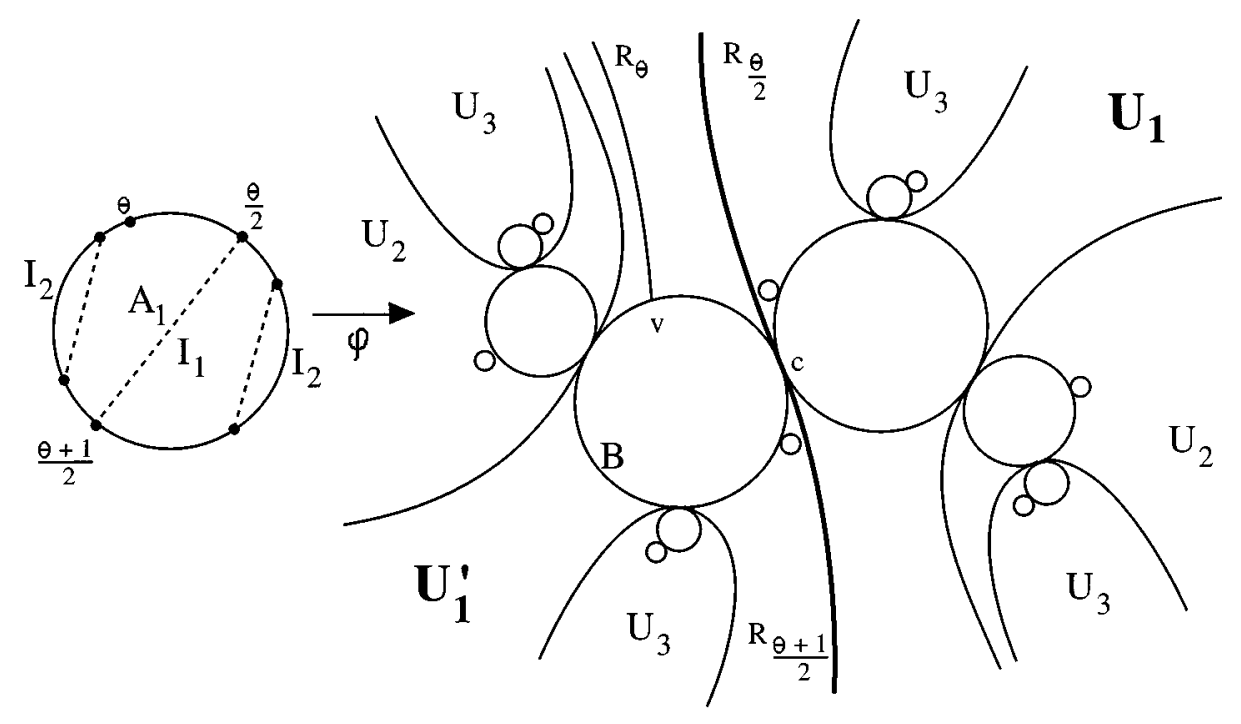

Figure 1. Locally connected Siegel Julia set

part of (3). Necessarily, $\theta$ is irrational, since otherwise the critical point would be preperiodic, and, therefore, $J(P)$ would have no bounded complementary domains $[8,34]$.

Under the map $P$, the rays $R_{\theta / 2}$ and $R_{(\theta+1) / 2}$ map onto the ray $R_{\theta}$, and, thus, they both land on the critical point $c$. Hence, $\bar{R}_{\theta / 2} \cup \bar{R}_{(\theta+1) / 2}$ separates the Riemann sphere into two open half-planes $U_{1}$ and $U_{1}^{\prime}$ with $v \in U_{1}^{\prime}$, such that $\left(\bar{U}_{1} \cap J\right) \cap$ $\left(\overline{U_{1}^{\prime}} \cap J\right)=\{c\}$. Note that $U_{1}$ and $U_{1}^{\prime}$ are unbounded simply connected domains whose boundary meets $J$ only at $c$. We will call such a domain a wedge with vertex the common point of the two boundary rays. See Figure 1.

Since $c \in B$, and $B$ is invariant, the forward orbit of $c$ remains in $B$, so in $U_{1}^{\prime}$. The forward orbit of the external ray $R_{\theta}$ consists of rays landing on the points of the forward orbit of $c$ on $B$. Hence, this orbit has to remain completely in the wedge $U_{1}^{\prime}$. Consequently, the forward orbit of $\theta$ remains in the half-circle $A_{1}=\left[\frac{\theta}{2}, \theta, \frac{\theta+1}{2}\right] \subset \mathbb{T}$. Let $I_{1}=\mathbb{T} \backslash A_{1}$. We have already observed that $\theta$ is irrational. From these two conditions, it follows (see Section 3.5) that

$$
\begin{gathered}
A=\mathbb{T} \backslash \bigcup_{i=0}^{\infty} \sigma^{-i}\left(I_{1}\right) \text { is a Cantor set, } \\
A \subset\left[\frac{\theta}{2}, \theta, \frac{\theta+1}{2}\right], \\
A=\overline{\mathcal{O}(\theta)}, \\
\left\{\frac{\theta}{2}, \frac{\theta+1}{2}\right\} \subset A .
\end{gathered}
$$

This establishes conclusions (4) and (5).

There is an intimate connection between $A$ and the building block $B$ moderated by the external rays corresponding to angles in $A$. Just as removing the open interval $I_{1}$ from $\mathbb{T}$ leaves behind the closed interval $A_{1}$ containing $A$, so removing the wedge $U_{1}$ from $\mathbb{C}$ leaves behind a closed wedge $B_{1}=\mathbb{C} \backslash U_{1}=\overline{U_{1}^{\prime}}$ containing a continuum $J_{1}=B_{1} \cap J$ containing $B$. Now, $\sigma^{-i}\left(I_{1}\right)$ consists of $2^{i}$ pairwise disjoint open subintervals, half of which lie in $\operatorname{Int}\left(A_{1}\right)$. It is successively deleting 
them that leaves a Cantor set $A$ behind. We call each of $U_{1}^{\prime}$ and $U_{1}$ a wedge of depth 0 . Correspondingly, $P^{-i}\left(U_{1}\right)$ consists of $2^{i}$ pairwise disjoint wedges of depth $i$ (between pairs of rays landing on preimages of the critical point $c$ ), in one-to-one correspondence (induced by the uniformization $\phi$ ) with the intervals in $\sigma^{-i}\left(I_{1}\right)$, half of which are contained in $\operatorname{Int}\left(B_{1}\right)=U_{1}^{\prime}$. For $i>1$, let $B_{i}=B_{i-1} \backslash P^{-i}\left(U_{1}\right)$. Let $J_{i}=B_{i} \cap J$ and let $B^{\prime}=\bigcap_{i=1}^{\infty} J_{i}$.

Since $B \subset J_{i}$ for all $i \geq 1$, we have that $B \subset B^{\prime}$. On the other hand, if $z \in B^{\prime}$, then some external ray $R_{\eta}$, with $\eta \notin \sigma^{-i}\left(I_{1}\right)$ for any $i$, lands on $z$. Thus, $\eta \in A$. There is a sequence $\left\{\eta_{i}\right\}_{i=1}^{\infty}$ in $A$ such that $\eta_{i} \rightarrow \eta$ and $R_{\eta_{i}}$ lands on a preimage of $c$ in $B$. It follows by Theorem 1.3 that $R_{\eta}$ lands on $B$. Hence, $z \in B$, so $B=B^{\prime}$. From this construction one can prove the following claims:

Every point of $B$ is the landing point of at most two rays

with arguments in $A$.

(6) For every angle in $A$ the corresponding ray lands on some point of $B$.

$$
\text { Two "endpoint" rays of } A \text { land on each point of } \mathcal{O}^{-}(c) \cap B \text {. }
$$

Exactly one "nonendpoint" ray lands on each point of $B \backslash \mathcal{O}^{-}(c)$.

Moreover, one can show that the uniformization $\phi$, extended to carry $\mathbb{T}$ onto $J$, when restricted to $A$, carries $A$ onto $B$, at most is two-to-one, and is circular-orderpreserving. Recalling that $\sigma: \mathbb{T} \rightarrow \mathbb{T}$ denotes the map $t \rightarrow 2 t(\bmod 1)$, we see directly that $\left.\sigma\right|_{A}: A \rightarrow A$ has the rotation number $\alpha$ when extended in the obvious way to a degree 1 order-preserving map of $\mathbb{T}$. That is, $B$ rotates "from the outside" the same as it does "from the inside." (See Section 3.5 for a discussion of rotation numbers of subsets of $\mathbb{T}$.) This proves conclusions (6) and (7).

To establish conclusions (8) and (9) of the theorem, we observe that starting with the Jordan curve $B$ and the two rays landing on $c \in B$ defining the two wedges in $\mathbb{C}_{\infty}$ with $B$ in one of them, we can reconstruct $J$ by "reflecting" $B$ (via $P^{-1}$ ) across $c$ into the other wedge. Then we can copy $B$ (via $P^{-2}$ ) into each of the wedges whose vertices are the two preimages of $c$ (one of which is in $B$ and one of which is in $B$ 's first reflection). Continuing this process ad infinitum, we construct a skeleton of $J$ : a countable union $S=\bigcup_{j=0}^{\infty} P^{-j}(B)$ of copies of $B$ meeting only at $c$ and preimages of $c$. This implies that

$$
\text { No external ray } R_{s} \text { with } s \in \mathbb{T} \backslash A \text { can land on } B \text {. }
$$

Note that $S$ is an $F_{\sigma}$ set in $J$, and dense, since $F$ contains a full inverse orbit. Recalling that any open subset of a Julia set eventually maps onto the Julia set, we see that $B$ has no interior in $J$, for it is forward invariant and clearly not all of $J$. Therefore, $S$ has no interior in $J$, since $J$ is a Baire space. We claim that

$$
G=J \backslash S \text { is a } 0 \text {-dimensional, dense } G_{\delta} \text { set in } J .
$$

Each point $z \in G$ is a limit point of copies of $B$ in a unique way.

For claim (10), that $G$ is a dense $G_{\delta}$ follows from the facts that $S$ is an $F_{\sigma}$ and $G$ is backward invariant. Claim (11) follows from the fact that each point $z \in G$ lies in a maximal tower of wedges. (By "maximal" we mean that no additional wedges can be inserted in the tower that contains $z$.) The existence of such a tower follows 
from the fact that

$$
G=\bigcap_{j=0}^{\infty}\left(P^{-j}\left(\bigcup_{i=0}^{\infty} P^{-i}\left(U_{1}\right)\right)\right) .
$$

By using the local connectivity of the Julia set, we can show that $z$ is the only point of $J$ in the intersection of its maximal tower of wedges, and that the tail of the tower, intersected with $J$, has diameter going to 0 . From this it follows that $G$ is 0-dimensional, finishing claim (10) and conclusion (8). Finally, the fact that $B$ is the boundary of the Siegel disk, on which $P$ is conjugate to the irrational rotation $r_{\alpha}$, proves that $B$, and consequently $S$, each point of which eventually maps into $B$, contains no (pre)periodic point of $P$, finishing conclusion (9).

To see the uniqueness part of conclusion (3), assume to the contrary that another external ray $R_{t}$ for $t \neq \theta$ also lands on the critical value $v$. Consider the wedge between $R_{t}$ and $R_{\theta}$ not containing $B$. The forward images of $R_{t}$ and $R_{\theta}$ must always land on $B$, but never on $c$. Since they are different external rays, there must be an open subset of $J$ in the wedge which never maps onto $B$, contradicting Sullivan's No Wandering Domains theorem. (Another way the uniqueness of $\theta$ can be seen to follow is from the one-to-one correspondence between invariant Cantor sets in $\mathbb{T}$ and irrational rotation numbers discussed in Section 3.5.)

2.2.1. Proof of Theorem 2.7. Let $P_{1}$ and $P_{2}$ be quadratic polynomials with critical points $c_{1}$ and $c_{2}$, invariant Siegel disks $\Delta_{1}$ and $\Delta_{2}$, and locally connected Julia sets $J_{1}$ and $J_{2}$, respectively. Let $B_{1}=\partial \Delta_{1}, B_{2}=\partial \Delta_{2}, S_{1}=\bigcup_{j=0}^{\infty} P_{1}^{-j}\left(B_{1}\right)$, $S_{2}=\bigcup_{j=0}^{\infty} P_{2}^{-j}\left(B_{2}\right)$ be the respective building blocks and skeleta from the proof of Theorem 2.10. The set of points of $B_{i}$ to which copies of $B_{i}$ are attached in forming $S_{i}$ is exactly the countable set $\mathcal{O}^{-}\left(c_{i}\right) \cap B_{i}$, dense in $B_{i}$ because $\left.P_{i}\right|_{B_{i}}$ is conjugate to some irrational rotation. It is easy to show that the pair $\left(B_{i}, \mathcal{O}^{-}\left(c_{i}\right) \cap B_{i}\right)$ is homeomorphic to the pair $(\mathbb{T}, \mathbb{Q} / \mathbb{Z})$. Hence, $\left(B_{1}, \mathcal{O}^{-}\left(c_{1}\right) \cap B_{1}\right)$ is homeomorphic to $\left(B_{2}, \mathcal{O}^{-}\left(c_{2}\right) \cap B_{2}\right)$. Since the respective skeleta are countable unions of copies of $B_{i}$ 's attached at points of $\mathcal{O}^{-}\left(c_{i}\right)$, one can extend this homeomorphism one copy at a time to a homeomorphism of $\left(S_{1}, \mathcal{O}^{-}\left(c_{1}\right)\right)$ onto $\left(S_{2}, \mathcal{O}^{-}\left(c_{2}\right)\right)$. From the facts that $J_{i} \backslash S_{i}$ is 0 -dimensional, any two distinct components of the complement of any copy of $B_{i}$ are disjoint, and the diameters of attached circles get smaller and smaller, it follows that the homeomorphism of $S_{1}$ onto $S_{2}$ extends uniquely to a homeomorphism of $J_{1}$ onto $J_{2}$.

Remark. The reader is cautioned that the above homeomorphism does not conjugate $P_{1}$ to $P_{2}$. The Julia sets are topologically equivalent, and have the same $F_{\sigma}$ skeleton and complementary $G_{\delta}$ "dust" structure, without necessarily being dynamically equivalent. Put another way, there is a single topological model $J$, the closure in $\mathbb{C}$ of a certain countable union of circles, and each irrational $\alpha$ of constant type induces a different dynamic on $J$ as the Julia set of a quadratic polynomial with irrationally indifferent fixed point of eigenvalue $e^{2 \pi i \alpha}$.

\section{Some subtleties}

3.1. Prime ends. Alternative, though equivalent, definitions of the impression of a prime end via "non-radial" rays and via crosscuts will be useful. For each $t \in \mathbb{T}$, there is some ray $\Sigma_{t}$ in $\mathbb{D}_{\infty}$, not necessarily radial, from $\infty$ to $t$ such that $S_{t}=\phi\left(\Sigma_{t}\right)$ 
has as its limit set $\operatorname{Im}\left(R_{t}\right)=\bar{S}_{t}-S_{t}$. While for $s \neq t, R_{s} \cap R_{t}=\emptyset$, the same cannot be said in general for the (nonradial) rays which converge to impressions.

Let $\phi: \mathbb{D}_{\infty} \rightarrow U_{\infty}$ be a uniformization carrying $\infty$ to $\infty$. Let $Q$ be an open arc in $U_{\infty}$ such that $\bar{Q} \cap \partial U_{\infty}=\{p, q\}$. Provided $p \neq q$, we call $Q$ a crosscut of $U_{\infty}$. Each crosscut $Q$ of $U_{\infty}$ separates $U_{\infty}$ into exactly two subdomains. Where well-defined, let $U(Q)$ denote the subdomain not containing $\infty$. A sequence $\left\{Q_{i}\right\}_{i=1}^{\infty}$ of crosscuts of $U_{\infty}$ is called a chain provided that for all $i$,

(1) $U\left(Q_{i+1}\right) \subset U\left(Q_{i}\right)$,

(2) for $j \neq i, \bar{Q}_{i} \cap \bar{Q}_{j}=\emptyset$, and

(3) $\phi^{-1}\left(Q_{i}\right) \rightarrow t$ for some $t \in \mathbb{T}$.

The relationship between chains of crosscuts and external rays is as follows. To each external ray $R_{t}$ there corresponds at least one chain $\left\{Q_{i}\right\}_{i=1}^{\infty}$ of crosscuts satisfying conditions (1)-(3) above such that $R_{t}$ meets each $Q_{i}$. Moreover, the chain can be chosen so that if $z \in \operatorname{Pr}\left(R_{t}\right)$, then $Q_{i} \rightarrow z$. Conversely, if $\left\{Q_{i}\right\}_{i=1}^{\infty}$ is a chain of crosscuts satisfying (1)-(3), then there is exactly one external ray $R_{t}$ such that $R_{t}$ meets each $Q_{i}$. We say that $\left\{Q_{i}\right\}_{i=1}^{\infty}$ defines the prime end $R_{t}$. Then the following holds:

$$
\operatorname{Im}\left(R_{t}\right)=\bigcap_{i=1}^{\infty} \overline{U\left(Q_{i}\right)}
$$

The reader familiar with prime ends will have noted that the usual definition of prime ends by means of equivalence classes of chains of crosscuts uses the following condition in place of (3) above:

$\left(3^{\prime}\right) Q_{i} \rightarrow z$ for some $z \in \partial U_{\infty}$.

Condition $\left(3^{\prime}\right)$ implies condition (3), though not conversely. However, the two definitions result in exactly the same prime end theory.

Let $Q_{i}$ be a crosscut of $U_{\infty}$ and $U\left(Q_{i}\right)$ the corresponding domain cut off. We define the shadow of $Q_{i}$ to be

$$
\operatorname{Sh}\left(Q_{i}\right)=\overline{U\left(Q_{i}\right)} \cap \partial U_{\infty}
$$

Suppose $\left\{Q_{i}\right\}_{i=1}^{\infty}$ defines the prime end $R_{t}$. An equivalent formulation of equation (13) is

$$
\operatorname{Im}\left(R_{t}\right)=\bigcap_{i=1}^{\infty} \operatorname{Sh}\left(Q_{i}\right)
$$

The following lemma is a corollary to Lemma 1.1 and equation (13):

Lemma 3.1. Let $\phi: \mathbb{D}_{\infty} \rightarrow U_{\infty}$ be a uniformization. Let $\left\{Q_{i}\right\}_{i=1}^{\infty}$ be a sequence of crosscuts of $U_{\infty}$ such that $\phi^{-1}\left(Q_{i}\right)$ converges to $t \in \mathbb{T}$. Then $\lim \sup \operatorname{Sh}\left(Q_{i}\right) \subset$ $\operatorname{Im}\left(R_{t}\right)$.

Proof. Note that $\left\{Q_{i}\right\}_{i=1}^{\infty}$ need not be a chain of crosscuts. However, since $\phi^{-1}\left(Q_{i}\right)$ $\rightarrow t$, any chain of crosscuts $\left\{P_{j}\right\}_{j=1}^{\infty}$ defining the prime end $R_{t}$ has the property that $U\left(P_{j}\right)$ contains all but finitely many $U\left(Q_{i}\right)$. Hence, $\lim \sup \operatorname{Sh}\left(Q_{i}\right) \subset \overline{U\left(P_{j}\right)}$ for every $j$. By (13), limsup $\operatorname{Sh}\left(Q_{i}\right) \subset \operatorname{Im}\left(R_{t}\right)$.

A priori, there are four possible outcomes in comparing the principal continuum of a prime end to its impression. Put in the language of external rays, prime ends can occur in any of the following mutually exclusive kinds: 
First kind: $\operatorname{Im}\left(R_{t}\right)=\operatorname{Pr}\left(R_{t}\right)$ is degenerate.

Second kind: Only $\operatorname{Im}\left(R_{t}\right)$ is nondegenerate.

Third kind: $\operatorname{Im}\left(R_{t}\right)=\operatorname{Pr}\left(R_{t}\right)$ is nondegenerate.

Fourth kind: $\operatorname{Im}\left(R_{t}\right)$ properly contains $\operatorname{Pr}\left(R_{t}\right)$, and both are nondegenerate.

Knowing what kinds of prime ends the complement of the filled-in Julia set has is a step in the direction of knowing the topology of the Julia set. For instance, the Julia set is locally connected iff all prime ends are of the first kind. (This is exactly when the uniformization can be extended to the boundary and the Carathéodory theorem applies.) Is the non-local-connectivity of Cremer and certain Siegel quadratic Julia sets the result of external rays which determine prime ends of the second, third, or fourth kind? We review what is known about this question in Section 3.3.

3.2. Julia sets and indecomposable continua. A continuum $X$ is decomposable iff it can be written as the union of two proper subcontinua, otherwise it is indecomposable. It is an open question whether or not the Julia set of any polynomial contains an indecomposable continuum. A theorem of Rutt's [29] allows one to identify indecomposable continua in the plane by means of prime ends. From it one can immediately draw some corollaries concerning Julia sets and external rays.

Theorem 3.2 (Rutt). Suppose $U$ is a simply connected plane domain with nondegenerate boundary. If $G$ is an indecomposable subcontinuum of $\partial U$, then there is a prime end $E$ such that $\operatorname{Im}(E) \supset G$. In particular, if $\partial U$ is indecomposable, then some prime end of $U$ has all of $\partial U$ as its impression.

Corollary 3.3. Suppose $P$ is a polynomial with connected Julia set $J=J(P)$. If $G$ is an indecomposable subcontinuum of $J$, then there is an external ray $R_{t}$ such that $\operatorname{Im}\left(R_{t}\right) \supset G$.

Corollary 3.4. Suppose $\Delta$ is a Siegel disk of a polynomial $P$ with connected Julia set $J=J(P)$. If $\partial \Delta$ is indecomposable, then there is an external ray $R_{s}$ such that $\operatorname{Im}\left(R_{s}\right) \supset \partial \Delta$.

In [19] there is more on the structure of indecomposable Julia sets, if there are any. Included are a corollary to Rutt's theorem, proved only for quadratic polynomials, and a converse to Rutt's theorem for all polynomials. It is an open question whether Corollary 3.5 works for polynomials of degree $d>2$.

Corollary 3.5 (Mayer/Rogers). Suppose $P$ is a quadratic polynomial with connected Julia set $J=J(P)$. If $J$ is an indecomposable continuum, then every external ray has as its impression all of $J$.

Theorem 3.6 (Mayer/Rogers). Suppose $P$ is a polynomial with connected Julia set $J=J(P)$. If $J$ is a decomposable continuum, then every external ray has impression nowhere dense in $J$.

3.3. Non-landing rays and Cremer points. That some external ray does not land is a sufficient condition for a polynomial Julia set not to be locally connected. Such a ray defines a prime end of the third or fourth kind. For non-local-connectivity however, it is enough to find a prime end of the second kind: one where the ray lands, so the principal continuum is an accessible point, but whose impression is nondegenerate. The first result about nonlanding rays was observed by Douady. Dan Sørensen provides a proof in his MS thesis [31]. Later, in his PhD thesis [32], 
Sørensen obtained further suggestive results on the structure of quadratic Julia sets where a nonlanding ray could be shown to exist.

Theorem 3.7 (Douady/Sørensen). For a generic set $\mathcal{G}$ of $\alpha \in \mathbb{T}$, there is a quadratic polynomial $P=P_{\alpha}$ with a Cremer fixed point of eigenvalue $e^{2 \pi i \alpha}$ such that there is an irrational $\theta=\theta(\alpha) \in \mathbb{T}$ with the critical value $v \in \operatorname{Pr}\left(R_{\theta}\right)$, and the rotation number of $\theta$ is the irrational $\alpha$. Moreover, the external ray $R_{\theta}$ does not land.

At the time the authors were preparing this paper, we became aware of related papers by two authors, each providing interesting details about the structure of Julia sets near irrationally indifferent fixed points. These papers contain much more than we report here. Jan Kiwi's results in [17] for Julia sets with a Cremer point were an inspiration for our Theorem 5.5, which presents a similar result for a quadratic Julia set with a Siegel disk not containing the critical point in its boundary.

Theorem 3.8 (Kiwi). Let $J$ be the Julia set of the polynomial $P$ with Cremer fixed point $z_{0}$ approximated by small cycles. Then there is a critical value $v \in J$ not accessible from $\mathbb{C}_{\infty} \backslash J$.

Theorem 3.9 (Kiwi). Let $J$ be the Julia set of the quadratic polynomial $P$ with Cremer fixed point $z_{0}$. Then the critical value $v \in J$ is not accessible from $\mathbb{C}_{\infty} \backslash J$. Indeed, if $v \in \operatorname{Pr}(R)$ (respectively, $v \in \operatorname{Im}(R)$ ) for some external ray $R$, then $z_{0} \in \operatorname{Pr}(R)$ (respectively, $z_{0} \in \operatorname{Im}(R)$ ).

We have modified Kiwi's statement of Theorem 3.9. Kiwi states the theorem only for $\operatorname{Pr}(R)$. A slight modification of his proof works for $\operatorname{Im}(R)$. This observation is useful because given any $z \in J$, there may be no ray whose principal continuum contains $z$, but there is always some external ray whose impression contains $z$.

There is a useful and interesting connection between Cremer points and periodic cycles. Yoccoz has proved that quadratic polynomials with a Cremer point have small periodic cycles converging to the Cremer point with periods $q_{n_{k}}$ for some subsequence of the denominators of the convergents of $\alpha$ (from the continued fraction expansion; see Section 1.1). Perez-Marco [22] has also proved that, for any polynomial, if $z_{0}$ is a Cremer point and $\alpha$ satisfies the condition

$$
\sum \frac{\log \log q_{n+1}}{q_{n}}<\infty,
$$

then there are small cycles converging to $z_{0}$ with periods as above.

In [24] (see also [23]) Perez-Marco proves the theorems below. In case there is a Siegel disk $\Delta$ at 0 with $\bar{\Delta} \subset U$, or in case 0 is a Cremer point, he calls the continuum $H$ of Theorem 3.10 a "hedgehog." This is apt, since he later shows that in these cases $\mathbb{C}_{\infty} \backslash H$ has many prime ends of the second kind.

Theorem 3.10 (Perez-Marco). Let $f(z)=e^{2 \pi i \alpha} z+O\left(z^{2}\right)$ be a holomorphic map defined on a simply connected neighborhood $U$ of 0 relatively compact in $\mathbb{C}$, and suppose that $f$ is univalent on a neighborhood of $\bar{U}$. Then there exists an $H \subset \mathbb{C}_{\infty}$ such that

1. $H$ is a cellular continuum $\left(\mathbb{C}_{\infty} \backslash H\right.$ is simply connected).

2. $0 \in H \subset \bar{U}$.

3. $H \cap \partial U \neq \emptyset$. 
4. $f(H)=H$.

5. $0 \in \partial H$ iff $f$ is nonlinearizable at 0 .

Moreover, if $h: \mathbb{C}_{\infty} \backslash \mathbb{D} \rightarrow \mathbb{C}_{\infty} \backslash H$ is a uniformization, then $g=h^{-1} f h$ extends continuously to an analytic circle diffeomorphism with rotation number $\alpha$.

Theorem 3.11 (Perez-Marco). In the setting of Theorem 3.10, let $H$ be a hedgehog and let $S$ denote $\partial \Delta$ or 0 , depending, respectively, upon whether or not $f$ is linearizable at 0 . Then one of the following two conditions holds:

1. $S$ is inaccessible from $\mathbb{C}_{\infty} \backslash H$.

2. For every prime end $E$ of $\mathbb{C}_{\infty} \backslash H$ such that $\operatorname{Pr}(E)$ is a single point $z$, all the points of $\operatorname{Im}(E) \backslash(\{z\} \cup S)$ are inaccessible.

Thus, $H$ will always contain inaccessible points. Moreover, each accessible point of $H \backslash S$ is the principal continuum of a prime end of the second kind.

In Theorems 4.1 and 4.3 , for a quadratic polynomial $P$ with an irrationally indifferent fixed point $z_{0}$, we prove the existence of an invariant "building block" continuum $B$ nowhere dense in the Julia set $J$. The critical point is in $B$, and, depending upon whether $P$ is linearizable at $z_{0}, B$ contains either the boundary of the Siegel disk about $z_{0}$ or the Cremer point $z_{0}$. What, one may ask, is the relationship between a hedgehog and a building block? If we add the Siegel disk to $B$, so the resulting continuum is cellular, the answer is that hedgehogs live in building blocks [24, Proposition I.3]. But, because the polynomial is not univalent on a neighborhood of the building block, the building block is larger than any hedgehog about $z_{0}$.

We understand that Shishikura has recently shown that a Julia set containing a Cremer point contains a set homeomorphic to a Cantor set cross an arc. This would be consistent with the corresponding building block $B$ being, or containing, a Cantor bouquet. (First cited in [11]. See [6] for the definition, and question (16) in Section 7.)

3.4. Siegel disks and their boundaries. Let $P$ be a polynomial of degree $d \geq 2$ with an irrationally indifferent periodic point $z_{0}$ of period $n$ such that $\left(P^{n}\right)^{\prime}\left(z_{0}\right)=$ $e^{2 \pi i \alpha}$. For simplicity, and without loss of generality, we may assume that $n=1$, otherwise, we may take as our function $P^{n}$, under which $z_{0}$ is a fixed point. Suppose that $z_{0}$ is a Siegel point; that is, there is an invariant Siegel disk $\Delta$ on which $P$ is conjugate to the rigid rotation $r_{\alpha}$ of the unit disk through angle $\alpha \in \mathbb{T}$. The following is a long-standing question of Douady and Sullivan [12, 13, 34]: Must $\partial \Delta$ always be a Jordan curve?

A construction of Douady [13], following work of Ghys, showed that the boundary was sometimes a Jordan curve. Petersen used this construction to prove his previously cited theorem where he shows the entire quadratic Julia set is locally connected provided $\alpha$ is of constant type. In this case the critical point is in $\partial \Delta$. Using a similar construction, M. Herman [16, Theorem 5] has examples of a quadratic polynomial of the form $f(z)=e^{2 \pi i \alpha}\left(z+z^{2}\right)$ with a Siegel disk $\Delta$ such that the whole orbit of the critical point is disjoint from $\partial \Delta$ (hence, with a non-locallyconnected Julia set). In all these cases $\partial \Delta$ is a quasi-circle, that is, the image of $S^{1}$ under a quasi-conformal homeomorphism.

If $\partial \Delta$ is a Jordan curve, then the conjugation to an irrational rotation extends to the boundary, as we have previously observed, and hence, $\partial \Delta$ contains no periodic points of $P$. Three related questions are evident: Is $\partial \Delta$ a Jordan curve? Is a 
critical point in $\partial \Delta$ ? Is a periodic point in $\partial \Delta$ ? Various partial answers have been obtained. One can expand the first question as follows: If $\partial \Delta$ is not a Jordan curve, is it a decomposable continuum, or is it an indecomposable continuum? That this is a useful question will be apparent from the Structure Theorem of J. T. Rogers which we discuss below.

A continuum $X$ is said to be irreducible if there are points $p$ and $q$ in $X$ such that no proper subcontinuum of $X$ contains $p$ and $q . X$ is said to be circularly irreducible if there exist two points $p$ and $q$ in $X$ such that $X=A \cup B$, where $A$ and $B$ are two subcontinua of $X$, irreducible between $p$ and $q$, and such that $A \cap B$ consists of two disjoint subcontinua of $A$ and $B$. K. Kuratowski has proved in [18] the following result:

Theorem 3.12 (Kuratowski). Let $X$ be a circularly irreducible continuum such that every indecomposable subcontinuum of $X$ is nowhere dense in $X$. Then there exists a monotone map $\phi: X \rightarrow \mathbb{T}$ such that for every $t \in \mathbb{T}, \phi^{-1}(t)$ is a nowhere dense subcontinuum of $X$, called the tranche at $t$.

Remark. E. Dyer has shown that if in addition the map $\phi$ is open, then a dense set of tranches are indecomposable subcontinua [14].

A continuum in $\mathbb{C}$ is said to be tree-like iff it is nowhere dense in $\mathbb{C}$ and does not separate $\mathbb{C}$. Every subcontinuum of a tree-like continuum is tree-like, and the intersection of any two subcontinua of a tree-like continuum is connected. Rogers proved the following Structure Theorem for the boundary of a Siegel disk $\Delta$ of a polynomial $P$.

Theorem 3.13 (Rogers [26]). If $\partial \Delta$ is decomposable, then the conformal map $\phi$ : $\Delta \rightarrow \mathbb{D}$ that results from the Riemann Mapping Theorem extends to a surjective mapping $\psi: \mathbb{C} \rightarrow \mathbb{R}^{2}$ in such a way that $\psi(\partial \Delta)=\partial \mathbb{D}=\mathbb{T}$ and $\left\{\psi^{-1}(t): t \in \mathbb{T}\right\}$ is an upper-semicontinuous decomposition of $\partial \Delta$ into tree-like subcontinua. Moreover,

1. the subcontinua $\left\{\psi^{-1}(t)\right\}_{t \in \mathbb{T}}$ are the impressions of the internal rays $R_{t}$,

2. $\left\{\psi^{-1}(t)\right\}_{t \in \mathbb{T}}$ are nowhere dense in $\partial \Delta$, and are accessible from inside $\Delta$ at no more than one point,

3. $\left.\psi\right|_{\mathbb{C} \backslash \bar{\Delta}}$ is one-to-one, and

4. for any nondegenerate proper interval $\left[s_{1}, s_{2}\right] \subset \mathbb{T}, \psi^{-1}\left(\left[s_{1}, s_{2}\right]\right)$ is a continuum which is irreducible between $\psi^{-1}\left(s_{1}\right)$ and $\psi^{-1}\left(s_{2}\right)$.

Remark. For $t \in \mathbb{T}$, let $T_{t}=\psi^{-1}(t)$. We call $T_{t}$ a tranche of the decomposition of $\Delta$ induced by $\psi$. If we consider the Riemann map $\phi$ to be the unique representation with $\phi\left(z_{0}\right)=0$ and $\phi^{\prime}\left(z_{0}\right) \in \mathbb{R}^{+}$, then the mapping $\psi \circ P \circ \psi^{-1}: \mathbb{R}^{2} \rightarrow \mathbb{R}^{2}$ is well-defined and equal to the rigid rotation $r_{\alpha}$ on $\overline{\mathbb{D}}$. Therefore, we have that $P\left(T_{t}\right)=T_{t+\alpha}$. Since $\alpha$ is irrational, there are no periodic continua (including points) of the polynomial $P$ on $\partial \Delta$ in the decomposable case. By Theorem 2.1, $\partial \Delta$ is an irreducible separator of $\mathbb{C}$. Hence, $\partial \Delta$ is circularly irreducible and every proper subcontinuum of $\partial \Delta$ is tree-like.

Independent proofs of the following theorem may be obtained without the assumption that $\partial \Delta$ is decomposable, either, following Rogers [28], by combining results of Herman, Poirier [15], and Rogers, or as a consequence of Perez-Marco [23, Theorem IV.4.2]. Rogers further observes that if $\partial \Delta$ contains a periodic point, then it must be indecomposable. 

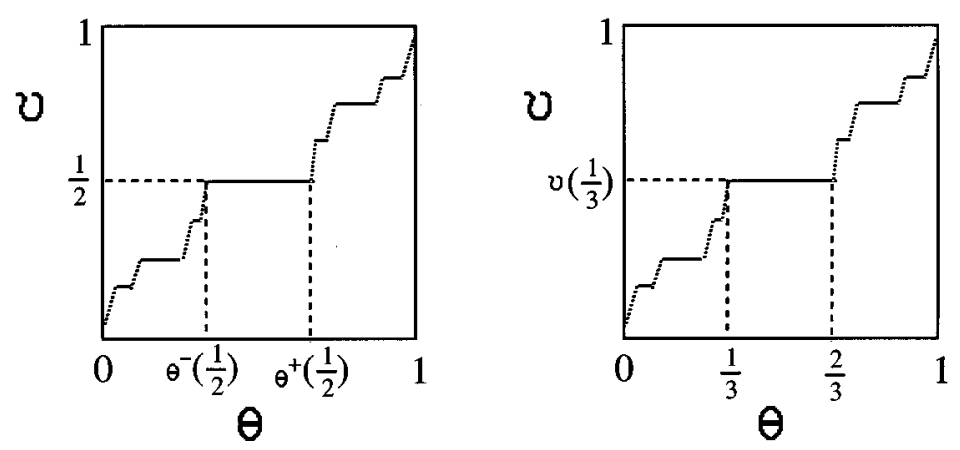

Figure 2. Devil's staircase

Theorem 3.14 (Perez-Marco, Rogers). Let $P$ be a polynomial with an invariant Siegel disk $\Delta$ such that there are no critical points in an open topological disk containing $\bar{\Delta}$. Then $\partial \Delta$ contains no periodic points.

3.5. The devil's staircase. Let $\sigma: A \rightarrow \mathbb{T}$ be the map $\sigma(t)=2 t$ from a subset $A$ of the circle, which is order preserving. Let $F: \mathbb{T} \rightarrow \mathbb{T}$ be an order-preserving extension of $\sigma$, and $\bar{F}: \mathbb{R} \rightarrow \mathbb{R}$ be the lift of $F$ satisfying $\bar{F}(y+1)=\bar{F}(y)+1$. Then there is always a well defined rotation number associated with the set $A$, denoted by $\nu_{A}$ and defined by

$$
\nu_{A}=\lim _{n \rightarrow \infty} \frac{\bar{t}_{n}-\bar{t}_{0}}{n}
$$

where $t_{n}=\sigma^{n}\left(t_{0}\right)$ for some point $t_{0} \in A$ and $\bar{t}_{0}$ is a lift of $t_{0}$, while $\bar{t}_{n}$ is the unique lift of $t_{n}$ such that $0 \leq \bar{t}_{n}-\bar{t}_{n-1}<1$. It is easy to see that the map $\sigma$ restricted to $A$ is order preserving if and only if $A$ is subset of a closed semicircle.

Let $\theta \in \mathbb{T}$ be a number, and let $A=\left\{t \in \mathbb{T} \mid \mathcal{O}_{\sigma}(t) \subset\left[\frac{\theta}{2}, \theta, \frac{\theta+1}{2}\right]\right\}$. Then $\left.\sigma\right|_{A}$ is order preserving and $A$ has a well defined rotation number $\nu_{A}$ which we will denote by $\nu(\theta)$ and we shall call it the rotation number of $\theta$. The rotation number $\nu(\theta)$ is given by the "devil's staircase" graph (see Figure 2) [7]. The following results combine various theorems in [7].

Theorem 3.15 (Bullett/Sentenac). Let $\theta \in \mathbb{T}$ be a number, and let $A=\{t \in$ $\left.\mathbb{T} \mid \mathcal{O}_{\sigma}(t) \subset\left[\frac{\theta}{2}, \theta, \frac{\theta+1}{2}\right]\right\}$. Then either

1. $\nu(\theta) \in \mathbb{Q} / \mathbb{Z}$, in which case $A$ is either a finite periodic orbit or a countably infinite set preperiodic to a periodic orbit; or

2. $\nu(\theta) \in(\mathbb{R} \backslash \mathbb{Q}) / \mathbb{Z}$, in which case $A$ is a Cantor set, contained in the semicircle $\left[\frac{\theta}{2}, \theta, \frac{\theta+1}{2}\right]$, unique with respect to being invariant under $\sigma$ with rotation number $\nu(\theta)$, and contains both end points of the semicircle.

Theorem 3.16 (Bullett/Sentenac). Let $\nu \in \mathbb{T}$. Then there exists a unique minimal closed invariant subset $A_{\nu} \subset \mathbb{T}$, with rotation number $\nu$. Moreover:

1. If $\nu \in \mathbb{Q}$, then $A_{\nu}$ is a periodic orbit and there exists a unique pair of rationals $0<\theta_{\nu}^{-}<\theta_{\nu}^{+}<1$ such that:

(a) $\theta_{\nu}^{-}$and $\theta_{\nu}^{+}$are both in $A_{\nu}$ and are adjacent on $\mathbb{T}$,

(b) $\left\{\theta_{\nu}^{+} / 2, \theta_{\nu}^{-} / 2+1 / 2\right\} \subset A_{\nu}$ and $A_{\nu} \subset\left[\theta_{\nu}^{+} / 2, \theta, \theta_{\nu}^{-} / 2+1 / 2\right]$,

(c) if $\nu=p / q$ in reduced form, $\theta_{\nu}^{+}-\theta_{\nu}^{-}=\frac{1}{2^{q}-1}$. 
2. If $\nu \in \mathbb{R} \backslash \mathbb{Q}$, then $A_{\nu}$ is a Cantor set and there exists a unique real number $\theta_{\nu}$ such that $A_{\nu} \subset\left[\theta_{\nu} / 2, \theta_{\nu}, \theta_{\nu} / 2+1 / 2\right],\left\{\theta_{\nu} / 2, \theta_{\nu} / 2+1 / 2\right\} \subset A_{\nu}$, and $A_{\nu}=$ $\overline{\mathcal{O}_{\sigma}\left(\theta_{\nu}\right)}$.

Corollary 3.17. Let $\theta$ be an irrational number such that its orbit under $\sigma$ is contained in the semicircle $\left[\frac{\theta}{2}, \theta, \frac{\theta+1}{2}\right]$. Then $A=\overline{\mathcal{O}_{\sigma}(\theta)}$ is a Cantor set, invariant under $\sigma$, containing both endpoints of the semicircle, unique with the above properties, and with rotation number $\nu(\theta)$ an irrational number.

\section{Statements of MAIN RESUlts}

Theorem 4.1 (Building Block Theorem, Siegel Case). Let $P$ be a quadratic polynomial with a linearizable fixed point $z_{0}$ with eigenvalue $e^{2 \pi i \alpha}$ and $\Delta$ the invariant Siegel disk about $z_{0}$, Julia set $J=J(P)$, and critical point c. Suppose there is no external ray $R_{s}$ such that $\partial \Delta \subset \operatorname{Im}\left(R_{s}\right)$. Then there is a building block $B \subset J$ and a unique Cantor set $A \subset \mathbb{T}$ such that the following hold:

1. $B$ is a nowhere dense subcontinuum of $J$ not contained in the impression of any external ray.

2. $P(B)=B$.

3. $\partial \Delta \subset B$.

4. $\mathcal{O}(c) \subset B$.

5. There is a unique $\theta \in \mathbb{T}$ such that $v=P(c) \in \operatorname{Im}\left(R_{\theta}\right)$.

6. $\mathcal{O}(\theta)$ is dense in $A$.

7. $A \subset\left[\frac{\theta}{2}, \theta, \frac{\theta+1}{2}\right]$.

8. $\sigma(A)=A$, minimally, with rotation number $\alpha$.

9. If $t \in A$, then $\operatorname{Im}\left(R_{t}\right) \subset B$ and $\operatorname{Im}\left(R_{t}\right) \cap \partial \Delta \neq \emptyset$.

10. If $z \in B$, then for some $t \in A, z \in \operatorname{Im}\left(R_{t}\right)$.

11. There are no periodic points in $B$.

Note that the assumption that no external ray's impression contains the boundary of the Siegel disk simultaneously rules out an indecomposable Julia set and an indecomposable Siegel disk boundary.

Corollary 4.2. In the setting of Theorem 4.1, there is a skeleton

$$
S=\bigcup_{n=0}^{\infty} P^{-n}(B)
$$

in $J$ such that the following hold:

1. $S$ is a dense $F_{\sigma}$ in $J$ with empty interior.

2. $S$ is fully invariant.

3. $S$ contains no periodic points.

4. $J \backslash S$ is a dense $G_{\delta}$, fully invariant under $P$, and contains all the periodic points in $J$.

Theorem 4.3 (Building Block Theorem, Cremer Case). Let $P$ be a quadratic polynomial with a Cremer fixed point $z_{0}$ with eigenvalue $e^{2 \pi i \alpha}$. Suppose further that the Julia set $J=J(P)$ is decomposable. Let $c$ denote the critical point of $P$. Then there is a building block $B \subset J$ and a Cantor set $A \subset \mathbb{T}$ such that the following hold:

1. $B$ is a nowhere dense subcontinuum of $J$.

2. $P(B)=B$. 
3. $P^{-1}\left(z_{0}\right) \subset B$.

4. $\mathcal{O}(c) \subset B$.

5. There is a $\theta \in \mathbb{T}$ such that $v=p(c) \in \operatorname{Im}\left(R_{\theta}\right)$.

6. $\mathcal{O}(\theta)$ is a dense subset of $A$.

7. $A \subset\left[\frac{\theta}{2}, \theta, \frac{\theta+1}{2}\right]$.

8. $\sigma(A)=A$, minimally, with rotation number $\alpha$.

9. If $t \in A$, then $\operatorname{Im}\left(R_{t}\right) \subset B$ and $z_{0} \in \operatorname{Im}\left(R_{t}\right)$.

10. If $z \in B$, then for some $t \in A, z \in \operatorname{Im}\left(R_{t}\right)$.

The assumption that the Julia set is decomposable is necessary to obtain that the impression containing $v$ is proper in $J$.

Corollary 4.4. In the setting of Theorem 4.3, there is a skeleton

$$
S=\bigcup_{n=0}^{\infty} P^{-n}(B)
$$

in $J$ such that the following hold:

1. $S$ is a dense $F_{\sigma}$ in $J$ with empty interior.

2. $S$ is fully invariant.

3. $J \backslash S$ is a dense $G_{\delta}$, fully invariant under $P$.

Remark. In the Cremer Building Block Theorem, the Cantor set $A$ should be unique and the building block $B$ should contain no periodic points, but we have not been able to prove this.

\section{Supporting LEMmas}

Most of the results in this section can also be proved without the assumption that the boundary of $\Delta$ is a decomposable continuum, as well as for polynomials of degree $d \geq 2$, by using the Milnor-Goldberg Fixed Point Portrait. Proofs of these will appear in a subsequent paper.

Lemma 5.1. Suppose $\Delta$ is an invariant Siegel disk of a quadratic polynomial $P$ such that $\partial \Delta$ is a decomposable continuum. Let $G$ be a proper subcontinuum of $J$. Then $G \cap \partial \Delta$ is either empty or a continuum.

Proof. Let $z_{0}$ be the indifferent (Siegel) fixed point such that $P^{\prime}\left(z_{0}\right)=e^{2 \pi i \alpha}$. By Theorem 3.13, there exists a monotone map $\psi: \mathbb{C} \rightarrow \mathbb{R}^{2}$ carrying $\partial \Delta$ to $\mathbb{T}$ such that $T_{s}=\psi^{-1}(s)$ is a tree-like continuum for each $s \in \mathbb{T}$ and $\psi$ is a homeomorphism off $\partial \Delta$.

Suppose that $\psi(G \cap \partial \Delta)$ is not connected. By the $\Theta$-curve theorem, there is an $s \in \mathbb{T}$ such that $s$ does not belong to the closure of the unbounded complementary domain of $\psi(G) \cup \mathbb{T}$. Hence, $T_{s} \cap \overline{U_{\infty}}=\emptyset$, a contradiction. Hence, we may assume $\psi(G \cap \partial \Delta)$ is connected. Then $\psi(G \cap \partial \Delta)=\left[s_{1}, s_{2}\right]$, an interval in $\mathbb{T}$, where $s_{1}=s_{2}$ if either $\psi(G \cap \partial \Delta)=\mathbb{T}$ or $\psi(G \cap \partial \Delta)=\left\{s_{1}\right\}$. Let $\left(s_{1}, s_{2}\right)=\psi(G \cap \partial \Delta) \backslash\left\{s_{1}, s_{2}\right\}$. There are then two cases.

Case 1. Suppose that for all $s \in\left(s_{1}, s_{2}\right)$, we have $T_{s} \subset G$. Set

$$
K=\overline{\bigcup_{s \in\left(s_{1}, s_{2}\right)} T_{s}}
$$


Then $K$ is a continuum (empty if $\psi(G \cap \partial \Delta)=\left\{s_{1}\right\}$ ) and

$$
\partial \Delta \cap G=\left(T_{s_{1}} \cap G\right) \cup K \cup\left(T_{s_{2}} \cap G\right) .
$$

If $s_{1}=s_{2}$ and $\left(s_{1}, s_{2}\right)=\mathbb{T} \backslash\left\{s_{1}\right\}$, since tranches are nowhere dense by Theorem 3.13, it follows that $T_{s_{1}} \subset K$, and thus $\partial \Delta \cap G=\partial \Delta$. Hence, we may assume that either $s_{1} \neq s_{2}$ (so $\left[s_{1}, s_{2}\right]$ is a proper nondegenerate subinterval of $\mathbb{T}$ ), or $s_{1}=s_{2}=$ $\psi(\partial \Delta \cap G)$. If $T_{s_{i}} \cap G$ is connected, for $i=1,2$, then $\partial \Delta \cap G$ is connected given either alternative.

Therefore, without loss of generality, we may suppose that $T_{s_{1}} \cap G=A \cup B$, where $A$ and $B$ are disjoint closed nonempty sets, and that $\psi(\partial \Delta \cap G)$ is a proper (possibly degenerate) subinterval of $\mathbb{T}$. Then $T_{s_{1}} \cup G$ separates the plane, and there exists a bounded complementary domain $V$ of $T_{s_{1}} \cup G$ such that $\partial V \backslash G \neq \emptyset$. Since $\partial V \subset T_{s_{1}} \cup G$ and $\partial V \backslash G \subset T_{s_{1}}$, it follows that $V \cap \Delta=\emptyset$. Since $V \cap \overline{U_{\infty}}=\emptyset, V$ must be a bounded complementary domain of $J$.

By Sullivan's No Wandering Domains Theorem [34], each bounded Fatou component is preperiodic. Since $P$ is quadratic, all bounded Fatou components are preperiodic to the fixed Siegel disk $\Delta$. Hence, there exists a minimal $n>0$ such that $P^{n}(V)=\Delta$. Choose rays $R_{i}, i \in\left\{1,2, \ldots, 2^{n+1}\right\}$, in $V$ landing in $T_{s_{1}} \backslash G$. Since $P^{n}$ is at most $2^{n}$ to one, $P^{n}\left(R_{i}\right)$ are rays landing on at least two distinct points in $P^{n}\left(T_{s}\right)=T_{s+n \alpha}$, which contradicts the fact that, by Theorem 3.13, each tranche of $\partial \Delta$ is accessible from $\Delta$ in at most one point.

Case 2. Suppose there exists an $s \in\left(s_{1}, s_{2}\right)$ such that $T_{s} \backslash G \neq \emptyset$. Since tranches are nowhere dense in $\partial \Delta$, there exist three distinct points $a, b, c \in \mathbb{T}$ such that $b \in[a, c] \subset\left(s_{1}, s_{2}\right)$ and $T_{b} \backslash G \neq \emptyset$. Let $B$ be the interval in $\mathbb{T}$ with endpoints $a$ and $c$ that does not contain $b$. Set $K=\psi^{-1}(B) \cup G$. Then $K$ is a continuum which separates the plane. Since $G \cap \Delta=\emptyset$ and $G$ meets both $T_{a}$ and $T_{c}$, and since $\psi^{-1}(B)$ is an arc of tranches from $T_{a}$ to $T_{c}, \Delta$ must be contained in a bounded complementary domain $E$ of $K$, bordered in part by tranches corresponding to points in $B$. Meanwhile, $U_{\infty}$ is contained in the unbounded complementary domain of $K$. Choose $x \in T_{b} \backslash G$. Since $x \in \bar{\Delta}$ and $\Delta \subset E, x \in E$. This contradicts $x \in \overline{U_{\infty}}$.

Remark. Suppose that $\partial \Delta$ is a decomposable continuum. Let $R_{s}$ be an external ray and let $K_{s}=\operatorname{Im}\left(R_{s}\right) \cap \partial \Delta$. Given that $K_{s} \neq \partial \Delta$, it seems very unlikely that $K_{s}$ could hit more than one tranche, but we have been unable to prove that.

Corollary 5.2. Suppose $\Delta$ is an invariant Siegel disk of a quadratic polynomial $P$ such that $\partial \Delta$ is a decomposable continuum. For each external ray $R_{s}, K_{s}=$ $\operatorname{Im}\left(R_{s}\right) \cap \partial \Delta$ is either empty or a continuum. Moreover, for all $n \geq 1, P^{n}\left(K_{s}\right) \subset$ $K_{2^{n} s}$.

Proof. Observe that

$$
P^{n}\left(K_{s}\right)=P^{n}\left(\partial \Delta \cap \operatorname{Im}\left(R_{s}\right)\right) \subset \partial \Delta \cap P^{n}\left(\operatorname{Im}\left(R_{s}\right)\right)=\partial \Delta \cap \operatorname{Im}\left(R_{2^{n} s}\right)=K_{2^{n} s} .
$$

Lemma 5.3. Let $P$ be a polynomial of degree $d \geq 2$ and $\Delta$ an invariant Siegel disk of $P$ with decomposable boundary. Then $\partial \Delta$ does not contain a proper subcontinuum invariant under $P^{n}$. 
Proof. This lemma follows immediately from the observation that the monotone map $\psi: \partial \Delta \rightarrow \mathbb{T}$ semiconjugates $\left.P\right|_{\partial \Delta}$ with an irrational rotation on $\mathbb{T}$. (See the remark after Theorem 3.13.)

Lemma 5.4. Suppose $\Delta$ is an invariant Siegel disk of a quadratic polynomial $P$ such that $\partial \Delta$ is a decomposable continuum. Let $R_{t}$ be an external ray whose impression meets $\partial \Delta$ properly. Then $t$ is an irrational number.

Proof. Suppose, on the contrary, that there exists some $t \in \mathbb{Q}$ such that $\operatorname{Im}\left(R_{t}\right)$ meets $\partial \Delta$ properly. By Corollary $5.2, K_{t}=\operatorname{Im}\left(R_{t}\right) \cap \partial \Delta$ is a proper nonempty subcontinuum of $\partial \Delta$. Since $\mathrm{t}$ is a rational number, there exist positive integers $m$ and $n$ such that

$$
P^{m}\left(R_{2^{n} t}\right)=R_{2^{n} t},
$$

which implies that $P^{m}\left(K_{2^{n} t}\right) \subset K_{2^{n} t}$. Hence, $K_{2^{n} t}$ is a proper invariant subcontinuum of $\partial \Delta$, contradicting Lemma 5.3.

Theorem 5.5. Let $P$ be a quadratic polynomial with an invariant Siegel disk $\Delta$ that does not contain the critical value in its boundary. Then exactly one of the following happens:

1. There exists an external ray $R_{s}$ such that $\partial \Delta \subset \operatorname{Im}\left(R_{s}\right)$.

2. For any ray $R_{t}$ with the critical value $v \in \operatorname{Im}\left(R_{t}\right), \operatorname{Im}\left(R_{t}\right) \cap \partial \Delta$ is a proper nonempty subcontinuum of $\partial \Delta$.

Proof. Suppose that the first does not hold, that is, there is no external ray containing $\partial \Delta$ in its impression. By Corollary $3.4, \partial \Delta$ is decomposable. Let $R_{t}$ be an external ray with $v \in \operatorname{Im}\left(R_{t}\right)$ and suppose that $\operatorname{Im}\left(R_{t}\right)$ does not meet $\partial \Delta$. Let $U_{0}=\mathbb{C}_{\infty} \backslash \widehat{R}_{t}$, and notice that $\bar{\Delta} \subset U_{0}$. Since $\widehat{R}_{t / 2}$ and $\widehat{R}_{(1+t) / 2}$ both contain the critical point $c, \widehat{R}_{t / 2} \cup \widehat{R}_{(1+t) / 2}$ is a separator of $\mathbb{C}_{\infty}$. Hence, $P^{-1}\left(U_{0}\right)$ consists of two simply connected open sets, each of which maps conformally one-to-one onto $U_{0}$. Let $U_{1}$ be the component of $P^{-1}\left(U_{0}\right)$ containing $\Delta$. We remark that $P$ maps $\partial \Delta$ homeomorphically onto itself and the restriction of $P$ on $\partial \Delta$ has no periodic points (see Lemma 5.3). Let

$$
B=\left\{s \in \mathbb{T}: \operatorname{Im}\left(R_{s}\right) \cap \partial \Delta \neq \emptyset\right\} .
$$

It is clear that for each $s \in B, R_{s} \subset U_{1}$. Since $\left.P\right|_{U_{1}}$ is a conformal isomorphism and $\partial \Delta$ is invariant, the two-shift map $\sigma: B \rightarrow B$, defined by $\sigma(s)=2 s$, is a homeomorphism. We shall prove that $B$ is a compact subset of $\mathbb{T}$. For this, let $\left(s_{n}\right)_{n \geq 1}$ be a sequence in $B$ converging to $s_{0}$. Then $\operatorname{Im}\left(R_{s_{n}}\right) \cap \partial \Delta \neq \emptyset$ for each positive integer $n$. By Lemma $1.1, \operatorname{Im}\left(R_{s_{0}}\right) \cap \partial \Delta \neq \emptyset$, so $s_{0} \in B$. By Corollary 2.4, since $\sigma$ is an expansive homeomorphism on the compact set $B$, we have that $B$ is finite. This contradicts Lemma 5.4.

Corollary 5.6. Let $P$ be a quadratic polynomial with an invariant Siegel disk $\Delta$ such that no external ray contains $\partial \Delta$ in its impression. Suppose that $R_{t}$ is an external ray containing the critical value $v$ in its impression. Then $\operatorname{Im}\left(R_{t}\right) \cap \partial \Delta$ is a nonempty proper subcontinuum of $\partial \Delta$.

Proof. If $v \notin \partial \Delta$, this is an immediate consequence of Theorem 5.5. If $v \in \partial \Delta$, it follows from Corollary 5.2. 
Lemma 5.7. Let $P$ be a polynomial of degree $d \geq 2$ with connected Julia set $J=J(P)$. Suppose that $R$ and $S$ are two external rays of $J$ such that $\operatorname{Im}(R) \cap \operatorname{Im}(S) \neq$ $\emptyset$, and let $U$ be an unbounded component of $\mathbb{C}_{\infty} \backslash(\widehat{R} \cup \widehat{S})$ and $T \subset \bar{U}$ an external ray. Then $\operatorname{Im}(T) \subset U \cup \operatorname{Im}(R) \cup \operatorname{Im}(S)$.

Proof. Note that $\widehat{R} \cup \widehat{S}$ is a separator of $\mathbb{C}_{\infty}$ and $\bar{U} \subset U \cup \widehat{R} \cup \widehat{S}$. We may assume that $T$ is an external ray in $U$. Let $z \in \operatorname{Im}(T)$ and let $\phi: \mathbb{D}_{\infty} \rightarrow U_{\infty}$ be the canonical Bötkher uniformization. Let $\phi^{-1}(T)$ land on $t \in \mathbb{T}$. Then there exists $w_{i} \in \mathbb{D}_{\infty}$ such that $w_{i} \in \phi^{-1}(U), w_{i} \rightarrow t$ and $\phi\left(w_{i}\right)$ converges to $z$. Hence,

$$
\operatorname{Im}(T) \subset \bar{U} \subset U \cup \widehat{R} \cup \widehat{S} .
$$

Lemma 5.8. Let $P$ be a quadratic polynomial with invariant Siegel disk $\Delta$ such that no external ray contains $\partial \Delta$ in its impression. Suppose that $R_{t}$ is an external ray containing the critical value $v$ in its impression. Then the forward orbit of $\widehat{R}_{t}$ is contained in the closure of the unbounded component of $\mathbb{C} \backslash\left(\widehat{R}_{t / 2} \cup \widehat{R}_{(t+1) / 2}\right)$ that contains $R_{t}$.

Proof. For $s \in \mathbb{T}$, let $K_{s}=\operatorname{Im}\left(R_{s}\right) \cap \partial \Delta$. By Corollary 5.2, if $K_{s} \neq \emptyset$, then $K_{s}$ is a proper subcontinuum of $\partial \Delta$, and for all $n \geq 1, P^{n}\left(K_{s}\right) \subset K_{2^{n} s}$. Since $\operatorname{Im}\left(R_{t / 2}\right)$ and $\operatorname{Im}\left(R_{(t+1) / 2}\right)$ both contain the critical point $c, \widehat{R}_{t / 2} \cup \widehat{R}_{(t+1) / 2}$ separates the plane into two unbounded complementary domains $U_{1}$ and $U_{2}$. For, if $\operatorname{Im}\left(R_{t / 2}\right) \cup \operatorname{Im}\left(R_{(t+1) / 2}\right)$ had a bounded complementary domain $V$, then by Sullivan's No Wandering Domains Theorem, there would be an $n$ such that $P^{n}(V)=\Delta$. Thus,

$$
\partial \Delta=P^{n}(\partial V) \subset P^{n}\left(\operatorname{Im}\left(R_{t / 2}\right) \cup \operatorname{Im}\left(R_{(t+1) / 2}\right)\right) \subset P^{n-1}\left(\operatorname{Im}\left(R_{t}\right)\right)=\operatorname{Im}\left(R_{2^{n-1} t}\right),
$$

contradicting our hypothesis.

Let $U_{1}$ denote the domain containing the external ray $R_{t}$ and $U_{2}$ the domain containing $R_{0}$ (corresponding to the two semicircles $[t / 2, t,(t+1) / 2]$ and $[(t+1) / 2,0, t / 2]$ of external rays $)$. By Corollary 5.6, $K_{t}=\partial \Delta \cap \operatorname{Im}\left(R_{t}\right) \neq \emptyset$. We claim that $\Delta \subset U_{1}$. Suppose, on the contrary, that $\Delta \subset U_{2}$. Then $\partial \Delta \cap U_{1}=\emptyset$, and hence, by Lemma 5.7,

$$
K_{t} \subset\left[\partial \Delta \cap \operatorname{Im}\left(R_{t / 2}\right)\right] \cup\left[\partial \Delta \cap \operatorname{Im}\left(R_{(t+1) / 2}\right)\right] .
$$

Thus, $K_{t} \subset K_{t / 2} \cup K_{(t+1) / 2}$, which implies that $P\left(K_{t}\right) \subset K_{t}$, a contradiction of Lemma 5.3.

Suppose, contrary to the statement of the theorem, that there exists some positive integer $m$ such that $R_{2^{m} t}$ belongs to the unbounded component $U_{2}$ of $\mathbb{C}_{\infty} \backslash\left(\widehat{R}_{t / 2} \cup \widehat{R}_{(t+1) / 2}\right)$ that does not contain $R_{t}$. Since $\Delta \subset U_{1}$, we have $\partial \Delta \cap U_{2}=\emptyset$. This implies, by Lemma 5.7, that

$$
\partial \Delta \cap \operatorname{Im}\left(R_{2^{m} t}\right) \subset\left[\partial \Delta \cap \operatorname{Im}\left(R_{t / 2}\right)\right] \cup\left[\partial \Delta \cap \operatorname{Im}\left(R_{(t+1) / 2}\right)\right] .
$$

Hence, $P^{m}\left(K_{t}\right) \subset K_{2^{m} t} \subset K_{t / 2} \cup K_{(t+1) / 2}$. Applying $P$, we have $P^{m+1}\left(K_{t}\right) \subset$ $P\left(K_{t / 2} \cup K_{(t+1) / 2}\right) \subset K_{t}$, contradicting Lemma 5.3. 


\section{Proof OF THE MAIN THEOREMS}

6.1. The Siegel disk case. We prove the Building Block Theorem 4.1 in the case of a quadratic polynomial $P$ with an invariant Siegel disk $\Delta$ such that no external ray contains $\partial \Delta$ in its impression. Since the only critical point is in the Julia set $J=J(P), J$ is a continuum. Since there is no external ray $R_{s}$ such that $\partial \Delta \subset \operatorname{Im}\left(R_{s}\right)$, we know by Corollaries 3.5 and 3.4 that both $J$ and $\partial \Delta$ are decomposable continua. We take up the cases of (1) a decomposable Julia set with an indecomposable Siegel disk boundary, (2) polynomials of degree $d>2$, and (3) periodic Siegel disks, in a subsequent paper.

6.1.1. Proof of Building Block Theorem 4.1. Since $J$ is a decomposable continuum, by Theorem 3.6 we know that for every external ray, its impression is nowhere dense in $J$. Since every point in $J$ must be in some impression, there is some $\theta \in \mathbb{T}$ such that for the critical value $v=P(c) \in J$, we have $v \in \operatorname{Im}\left(R_{\theta}\right)$.

By Corollary 5.6, $K_{\theta}=\operatorname{Im}\left(R_{\theta}\right) \cap \partial \Delta$ is a nonempty proper subcontinuum of $\partial \Delta$. By Lemma 5.4, $\theta$ is an irrational number in $\mathbb{T}$. Recall that $\widehat{R}_{\theta}=R_{\theta} \cup \operatorname{Im}\left(R_{\theta}\right)$. Let $U_{0}=\mathbb{C} \backslash \widehat{R}_{\theta}$. Observe that

$$
c \in \operatorname{Im}\left(R_{\frac{\theta}{2}}\right) \cap \operatorname{Im}\left(R_{\frac{\theta+1}{2}}\right) .
$$

It follows that $\widehat{R}_{\frac{\theta}{2}} \cup \widehat{R}_{\frac{\theta+1}{2}}$ is connected. Hence,

$$
\mathbb{C} \backslash P^{-1}\left(\widehat{R}_{\theta}\right)=\mathbb{C} \backslash\left(\widehat{R}_{\frac{\theta}{2}} \cup \widehat{R}_{\frac{\theta+1}{2}}\right)
$$

has exactly two unbounded components, each of which maps conformally isomorphically onto $U_{0}$ under $P$. Let $U_{1}^{\prime}$ be the unbounded component of $\mathbb{C} \backslash\left(\widehat{R}_{\frac{\theta}{2}} \cup \widehat{R}_{\frac{\theta+1}{2}}\right)$ that contains $R_{\theta}$, and $U_{1}$ the other unbounded component (which necessarily contains $\left.R_{0}\right)$.

We define the following four collections of subsets of $\mathbb{T}$ and $\mathbb{C}$ inductively:

$$
\begin{array}{rlrl}
I_{0}=\mathbb{T} \backslash\{\theta\}, & U_{0} & =\mathbb{C} \backslash \widehat{R}_{\theta}, \\
A_{0}=\bar{I}_{0}=\mathbb{T}, & B_{0} & =\bar{U}_{0}=\mathbb{C}, \\
I_{1}=\operatorname{component}\left(\sigma^{-1}\left(I_{0}\right), 0\right), & & =\operatorname{component}\left(P^{-1}\left(U_{0}\right), R_{0}\right), \\
A_{1} & =A_{0} \backslash I_{1}, & & B_{0} \backslash U_{1}, \\
& \vdots & & \vdots \\
I_{n}=\sigma_{n}^{-1}\left(I_{n-1}\right), & U_{n} & =P^{-1}\left(U_{n-1}\right), \\
A_{n}=A_{n-1} \backslash I_{n}, & \widehat{B} & =\bigcap_{n=0}^{\infty} \backslash U_{n}, \\
A & =\bigcap_{n=0}^{\infty} A_{n}, & & \\
& &
\end{array}
$$

We shall show that $A$ and $B$ satisfy conclusions (1)-(11) of the theorem. The reader may find it helpful to consult Figure 3 in what follows.

By Lemma 5.8 , the forward orbit of $\widehat{R}_{\theta}$ remains in $\overline{U_{1}^{\prime}}$. Hence, the forward orbit of $R_{\theta}$ never enters $U_{1}$, so the forward orbit of $\theta$ under $\sigma$ never enters $I_{1}$. This fact plus the irrationality of $\theta$ implies by Corollary 3.17 that $A$ is a Cantor set, which 


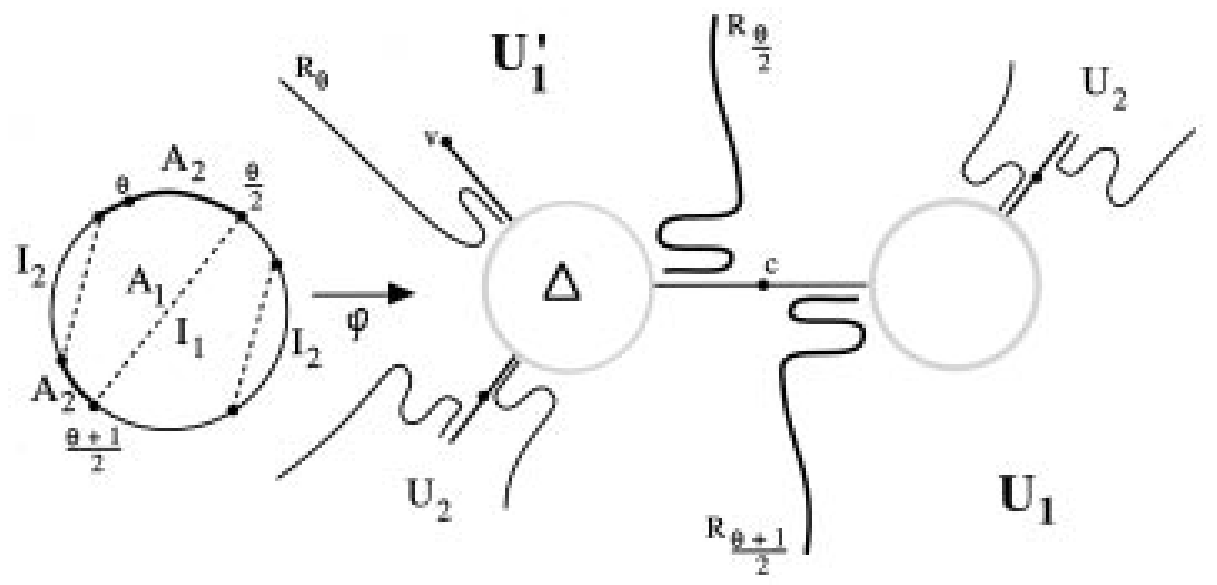

FiguRE 3. Building block construction for Siegel case

is the closure of the forward orbit of $\theta$, contained in the half-circle $A_{1}=\left[\frac{\theta}{2}, \theta, \frac{\theta+1}{2}\right]$, and contains both endpoints of $A_{1}$. In Theorem 6.1 below we prove that both $\theta$ and $A$ are unique and $A$ has the rotation number $\alpha$ under $\sigma$. This establishes conclusions (5)-(8) of the theorem. We prove that conclusion (11) holds in Theorem 6.2 below.

It remains to establish the properties of the building block $B$ and the relationship of $B$ to $A$. The proof of conclusions (1)-(4), (9), and (10) is based upon the following series of claims (for all $n \geq 0$ ):

$$
\text { There are no bounded components of } \mathbb{C} \backslash\left(\widehat{R}_{\frac{\theta}{2}} \cup \widehat{R}_{\frac{\theta+1}{2}}\right) \text {. }
$$

$$
\begin{gathered}
B_{1}=\mathbb{C} \backslash U_{1}=\overline{U_{1}^{\prime}} . \\
\widehat{B}=\mathbb{C} \backslash \bigcup_{n=0}^{\infty} P^{-n}\left(U_{1}\right) . \\
\widehat{R}_{\theta} \subset B_{n} . \\
\operatorname{Im}\left(R_{\theta}\right) \subset B .
\end{gathered}
$$

$B$ is properly contained in $J$.

$B_{n} \cap J$ is a nonempty continuum.

$$
\begin{gathered}
P(\widehat{B})=\widehat{B} . \\
t \in A_{n} \Longrightarrow \widehat{R}_{t} \subset B_{n} . \\
z \in B_{n} \cap J \Longrightarrow \exists t \in A_{n}, z \in \operatorname{Im}\left(R_{t}\right) .
\end{gathered}
$$

Assuming the claims hold, then conclusion (2) follows from claim (22) and the fact that $J$ is fully invariant. Conclusion (4) follows from claim (19) and conclusion (2), since $v \in \operatorname{Im}\left(R_{\theta}\right)$. Conclusion (3) follows from conclusion (4) and the fact that $\partial \Delta \subset \overline{\mathcal{O}(c)}$. By claim (21), since the $B_{n}$ 's are towered, $B$ is a nonempty continuum in $J$. If $B$ had interior in $J$, its forward image would map onto $J$ eventually, contradicting conclusion (2) and claim (20). If $B$ were contained in some impression, so would $\partial \Delta$ by conclusion (3), contradicting our hypothesis. Hence, conclusion (1) holds. The first part of conclusion (9) follows from claim (23) by intersecting over $n$ and with $J$. Conclusion (10) follows from claim (24), and Lemma 1.1. If $t \in A$ then, by conclusion (6), there is a subsequence $\left\{\theta_{n}\right\}_{n=1}^{\infty}$ of 
$\mathcal{O}(\theta)$ which converges to $t$. Since $\operatorname{Im}\left(R_{\theta}\right)$ meets $\partial \Delta$, Lemma 1.2 implies $\operatorname{Im}\left(R_{\theta_{n}}\right)$ meets $\partial \Delta$ for every $n$. By Lemma $1.1, R_{t}$ meets $\partial \Delta$, establishing the second part of conclusion (9).

To see claim (15), suppose the contrary. If $\mathbb{C} \backslash\left(\widehat{R}_{\frac{\theta}{2}} \cup \widehat{R}_{\frac{\theta+1}{2}}\right)$ contains a bounded domain $G$, then $G$ contains a bounded domain $H$ of $\mathbb{C} \backslash J$. By Sullivan's No Wandering Domains Theorem all bounded domains of $\mathbb{C} \backslash J$ are preperiodic to $\Delta$. Since every point of $\partial H$ is in $\bar{U}_{\infty}, \partial H \subset \operatorname{Im}\left(R_{\frac{\theta}{2}}\right) \cup \operatorname{Im}\left(R_{\frac{\theta+1}{2}}\right)$. But then $\partial P(H) \subset \operatorname{Im}\left(R_{\theta}\right)$. This implies some forward image of $\operatorname{Im}\left(R_{\theta}\right)$ contains $\partial \Delta$, contradicting our hypothesis. Claim (16) follows immediately. Claim (17) is an immediate consequence of the definitions of the $B_{n}$ 's and $\widehat{B}$.

For $n=0$, claim (18) is obvious. For $n=1$, we have already observed that $\widehat{R}_{\theta} \subset \overline{U_{1}^{\prime}}=B_{1}$. Since the forward orbit of $\theta$ remains in $A, \theta \in A_{n}$ for all $n \geq 0$. That is, $R_{\theta}$ is never in a component $W_{n}$ of $U_{n}$. By Lemma 5.7, $\widehat{R}_{\theta} \subset \mathbb{C} \backslash W_{n}$. By induction on $n$, and intersecting over the finitely many complements of components of $U_{n}$, we get $\widehat{R}_{\theta} \subset B_{n}$, establishing claim (18). Claim (19) now follows immediately by intersecting over $n$ and with $J$.

To see claim (20), recall that both $U_{1}$ and $U_{1}^{\prime}$ map isomorphically onto $U_{0}=$ $\mathbb{C} \backslash \widehat{R}_{\theta}$, and $\operatorname{Im}\left(R_{\theta}\right)$ is nowhere dense in $J$. Hence, both $U_{1}$ and $U_{1}^{\prime} \subset B_{1}$ contain nonempty open subsets of $J$ separated by $\operatorname{Im}\left(R_{\frac{\theta}{2}}\right) \cup \operatorname{Im}\left(R_{\frac{\theta+1}{2}}\right)$.

Claim (21) for $n=0$ is obvious. Claim (18) implies $B_{n} \cap J \neq \emptyset$ for all $n \geq 0$. Since $J$ is connected, both $\bar{U}_{1} \cap J$ and $\overline{U_{1}^{\prime}} \cap J=B_{1} \cap J$ are connected by the Boundary Bumping Theorem. Since $B_{1} \cap J$ is a continuum, and the components of $U_{2}$ are isomorphic preimages of $U_{1}$, we may repeat the argument with $U_{2}$ and $B_{2}=B_{1} \backslash U_{2}$ to show $B_{2} \cap J$ is connected. Claim (21) follows by induction.

To see claim (22), note that by claim (17), $P(\widehat{B}) \subset \widehat{B}$. If there were $z \in \widehat{B}$ with $z \notin P(\widehat{B})$, then $P^{-1}(z) \cap \widehat{B}=\emptyset$. If $P^{-1}(z)$ has a point in $U_{n}$ for some $n>1$, then $z \in U_{n-1}$. Hence, $z \notin \widehat{B}$, a contradiction. But $P^{-1}(z) \subset U_{1}$ contradicts the fact that $U_{1}$ contains at most one preimage of each point in $\mathbb{C}$. Thus, $P(\widehat{B})=\widehat{B}$.

To see claim (23), suppose $t \in A_{n}$. Then $t \notin I_{n}$, so $R_{t}$ is not in $U_{n}$. It follows that $R_{t} \subset B_{n}$. A proof similar to that for claim (18) shows that $\widehat{R}_{t} \subset B_{n}$.

To see claim (24), suppose $z \in B_{n} \cap J$ for some $n$. Note that

$$
B_{n}=\mathbb{C} \backslash \bigcup_{k=0}^{n} P^{-k}\left(U_{1}\right) \text {. }
$$

Let $W_{1}, \ldots, W_{m}$ be the components of $\mathbb{C} \backslash B_{n}$. Observe that each $W_{j}$ is a component of $P^{-i}\left(U_{1}\right)$ for some $i \in\{0, \ldots, n\}$. Let $t \in \mathbb{T}$ be such that $z \in \operatorname{Im}\left(R_{t}\right)$. Suppose there is no $j \in\{1, \ldots, m\}$ such that $R_{t} \subset \bar{W}_{j}$. Then $R_{t} \subset B_{n}$, so $t \in A_{n}$. On the contrary, suppose $R_{t} \subset \bar{W}_{j}$ for some $j \in\{1, \ldots, m\}$. Let $R_{u}$ and $R_{v}$ be the boundary rays of $W_{j}$. By Lemma $5.7, \operatorname{Im}\left(R_{t}\right) \subset W_{j} \cup \operatorname{Im}\left(R_{u}\right) \cup \operatorname{Im}\left(R_{v}\right)$. Since $z \notin W_{j}$, we have $z \in \operatorname{Im}\left(R_{u}\right) \cup \operatorname{Im}\left(R_{v}\right)$. Since $u, v \in A_{n}$, we are done.

Theorem 6.1. Let $P$ be a quadratic polynomial with an invariant Siegel disk $\Delta$ having rotation number $\alpha$ such that no external ray contains $\partial \Delta$ in its impression. Let $A \subset \mathbb{T}$ be the Cantor set generated by $\theta$, where the critical value $v \in \operatorname{Im}\left(R_{\theta}\right)$. Then the rotation number of $A$ is $\alpha$. In particular, $A$ and $\theta$ are unique.

Proof. Let $\psi: \mathbb{C} \rightarrow \mathbb{R}^{2}$ be the Rogers map (Theorem 3.13) which identifies each tranche of $\partial \Delta$ to a point and let $Q: \mathbb{R}^{2} \rightarrow \mathbb{R}^{2}$ be the map induced by $P$. For each 
$t \in \mathbb{T}$, let $L_{t}=\psi\left(K_{t}\right)$, where $K_{t}=\operatorname{Im}\left(R_{t}\right) \cap \partial \Delta$. Then $\psi(\partial \Delta)=S$ is a circle, $L_{t}$ is a possibly degenerate interval, and $\left.Q\right|_{S}=r_{\alpha}$ is rotation by $\alpha$. Compactify the plane $\mathbb{R}^{2}$ in a disk $\mathbb{D}$ such that each external ray $\psi\left(R_{t}\right)$ lands on the point $t \in \partial \mathbb{D}$. Extend the map $Q: \mathbb{R}^{2} \rightarrow \mathbb{R}^{2}$ to a map $\bar{Q}: \overline{\mathbb{D}} \rightarrow \overline{\mathbb{D}}$ by defining $\bar{Q}(t)=2 t(\bmod 1)$. Identify $\mathbb{R}^{2}$ with the interior of the open disk $\mathbb{D}$. Hence, $S=\psi(\partial \Delta)$ is a circle in the interior of $\mathbb{D}$ and $A \subset \partial \mathbb{D}$. If we identify components of the complement of $A$ in $\partial \mathbb{D}$ to a point we obtain a monotone map $m: \overline{\mathbb{D}} \rightarrow \overline{\mathbb{D}}$. For each $t \in A$, let $C_{t}=m\left(\psi\left(\hat{R}_{t}\right) \cup\{t\}\right)$. Then $C_{t}$ is a continuum joining the point $m(t) \in \partial \mathbb{D}$ to a proper subcontinuum $m\left(L_{t}\right)$ in $m(S)$, and $C_{t}$ is contained in the closed annulus $D$ bounded by $m(S)$ and $\partial \mathbb{D}$. Note that $\bar{Q}(\theta / 2)=\bar{Q}(\theta / 2+1 / 2)=\theta$. Hence, $\bigcup_{t \in A} C_{t}$ is invariant under $\bar{Q}$. Thus, $\bar{Q}$ induces a map $f$ on $\bigcup_{t \in A} C_{t}$. Note that $m(S) \cup \partial \mathbb{D} \subset \bigcup_{t \in A} C_{t}$. By definition, the rotation number of $A$ is the rotation number of the homeomorphism $\left.f\right|_{\partial \overline{\mathbb{D}}}: \partial \overline{\mathbb{D}} \rightarrow \partial \overline{\mathbb{D}}$. Note that $\left.f\right|_{m(S)}$ is a rotation by $\alpha$. By an argument similar to the proof of Lemma 5.7, for each $a, b, c>0, f^{b}\left(C_{\theta}\right)$ is contained in the closure of the component of $D \backslash\left(f^{a}\left(C_{\theta}\right) \cup f^{c}\left(C_{\theta}\right)\right)$ in the annulus $D$ containing the point $m\left(2^{b} \theta\right)$. Since $\left.f\right|_{m(S)}$ is rotation by $\alpha,\left.f\right|_{\partial \mathbb{D}}$ has rotation number $\alpha$.

Note that in the proof of the Building Block Theorem 4.1 we proved that $\theta$ always generates a Cantor set $A$ under the 2-shift map $\sigma$ lying in the semi-circle $\left[\frac{\theta}{2}, \theta, \frac{\theta+1}{2}\right]$. By Corollaries 5.6 and 5.2 , we know that $K_{\theta}$, is a subcontinuum of the boundary of the Siegel disk, while, by Lemma 5.4, we know that $\theta$ is irrational.

Suppose on the contrary, that there exist two irrational numbers $s_{1}$ and $s_{2}$ with $0<s_{1}<s_{2}<1$ such that the critical value $v=P(c)$ is contained in $\operatorname{both} \operatorname{Im}\left(R_{s_{1}}\right)$ and $\operatorname{Im}\left(R_{s_{2}}\right)$. As above, we know that $K_{s_{1}}=\partial \Delta \cap \operatorname{Im}\left(R_{s_{1}}\right)$ and $K_{s_{2}}=\partial \Delta \cap \operatorname{Im}\left(R_{s_{2}}\right)$ are proper subcontinua of $\partial \Delta$. Moreover, by hypothesis, $s_{1}$ and $s_{2}$ generate by the shift map $\sigma$ two Cantor sets $A_{1}$ and $A_{2}$ lying on the intervals $\left[\frac{s_{1}}{2}, s_{1}, \frac{1+s_{1}}{2}\right]$ and $\left[\frac{s_{2}}{2}, s_{2}, \frac{1+s_{2}}{2}\right]$ of $\mathbb{T}$, respectively. Notice, also, that since $A_{i}=\overline{\mathcal{O}_{\sigma}\left(s_{i}\right)}$, for $i=1,2$, the fact that $s_{1} \neq s_{2}$ implies that $A_{1} \cap A_{2}=\emptyset$. Since we have shown that the rotation numbers of $A_{1}$ and $A_{2}$ both must be $\alpha$, this contradicts Theorem 3.16. Hence, $\theta \in \mathbb{T}$ is such that $v \in \operatorname{Im}\left(R_{\theta}\right)$ is unique.

Theorem 6.2. Let $P$ be a quadratic polynomial with an invariant Siegel disk $\Delta$ such that no external ray contains $\partial \Delta$ in its impression. Let $B$ be the building block of Theorem 4.1 containing $\partial \Delta$. Then $B$ contains no periodic point of $P$.

Proof. Since there are no periodic points in $\partial \Delta$, suppose that there exists a point $z$ of period $n$ in $B \backslash \partial \Delta$, and let $R_{q}$ be an external ray with $q \in \mathbb{Q} / \mathbb{Z}$ landing at $z$. Suppose the critical value $v \in \operatorname{Im}\left(R_{\theta}\right)$. Since the Cantor set $A$ generated by $\theta$ is minimally invariant, it does not contain any rational number, and hence, $q \notin A$. Then $\frac{\theta+i}{2^{m}}<q<\frac{\theta+i+1}{2^{m}}$ for some $i, m \geq 0$. Since $R_{q}$ lands at $z$, and $z$ is not contained in the interior of any removed wedge, Lemma 5.7 implies that

$$
z \in \operatorname{Im}\left(R_{\frac{\theta+i}{2^{m}}}\right) \cup \operatorname{Im}\left(R_{\frac{\theta+i+1}{2^{m}}}\right),
$$

and, without loss of generality, $z \in \operatorname{Im}\left(R_{\frac{\theta+i}{2^{m}}}\right)$. This implies that

$$
P^{m}(z) \in \operatorname{Im}\left(P^{m}\left(R_{\frac{\theta+i}{2^{m}}}\right)\right)=\operatorname{Im}\left(R_{\theta}\right) .
$$

Since $z$ is periodic of period $n$, we may assume that $z \in \operatorname{Im}\left(R_{\theta}\right)$. Then $z=$ $P^{n}(z)=P^{2 n}(z)=\cdots=P^{k n}(z)=\ldots$ for each $k \in \mathbb{Z}^{+}$, which implies that $z \in \operatorname{Im}\left(R_{2^{k n} \theta}\right)$, for all $k \geq 0$. 
Consider the set $A_{0}=\left\{\theta, 2^{n} \theta, 2^{2 n} \theta, \ldots, 2^{k n} \theta, \ldots\right\} \subset A \subset \mathbb{T}$. By Lemma 1.1, $z \in \operatorname{Im}\left(R_{s}\right)$ for all $s \in \bar{A}_{0}$. Since the orbit of $\theta$ is dense in $A$ under $\sigma^{n}, \bar{A}_{0}=A$, and so $z \in \operatorname{Im}\left(R_{s}\right)$ for all $s \in A$. By Corollary 5.2 and Lemma 5.7, $K_{s} \subset K_{\theta / 2} \cup K_{\theta / 2+1 / 2}$ for all $s \in A$. Since $\partial \Delta \subset \bigcup_{s \in A} \operatorname{Im}\left(R_{s}\right)$, we have $\partial \Delta \subset K_{\frac{\theta}{2}} \cup K_{\frac{\theta+1}{2}}$. Therefore,

$$
\partial \Delta=P(\partial \Delta) \subset P\left(K_{\frac{\theta}{2}}\right) \cup P\left(K_{\frac{\theta+1}{2}}\right) \subset K_{\theta},
$$

which contradicts the hypothesis that there is no external ray containing $\partial \Delta$ in its impression.

6.1.2. Proof of Skeleton Corollary 4.2. We prove that the Siegel building block $B$ generates a skeleton $S=\bigcup_{n=0}^{\infty} P^{-n}(B)$ for the corresponding Julia set. Since $P^{n}$ is an open map and $B$ is nowhere dense and closed in $J, P^{-n}(B)$ is nowhere dense and closed. Thus, $S$ is an $F_{\sigma}$, and, since $J$ is a Baire space, $S$ has empty interior. However, since the inverse orbit of any point in $J$ is dense in $J, S$ is dense in $J$. By definition, $S$ is fully invariant. Since every point of $S$ eventually maps into $B$, any periodic points of $S$ must be in $B$. But $B$ contains no periodic points by Theorem 6.2. It follows that $J \backslash S$ contains all the periodic points of $J$. Clearly, $J \backslash S$ is a fully invariant dense $G_{\delta}$ in $J$.

One can say more: since $P: U_{1} \rightarrow U_{0}$ is a conformal isomorphism, one can pull $B \backslash\left(\operatorname{Im}\left(R_{\frac{\theta}{2}}\right) \cup \operatorname{Im}\left(R_{\frac{\theta+1}{2}}\right)\right)$ homeomorphically back into $U_{1}$. We see that $J$ contains two copies of $B$ joined along the "critical continuum" $C=\operatorname{Im}\left(R_{\frac{\theta}{2}}\right) \cup \operatorname{Im}\left(R_{\frac{\theta+1}{2}}\right)$. Let $W_{n}$ be a component of $U_{n}$. Since $P^{n-1}: W_{n} \rightarrow U_{1}$ is a conformal isomorphism, we see that $S$ can be viewed as the union of copies of $B$ joined along preimages of copies of $C$.

Just as $B$ is a union of impressions of external angles in the Cantor set $A, S$ is the union of impressions of external angles which eventually map into $A$. Consequently, by Theorem 6.1, $J \backslash S$ is contained in the union of impressions of external angles that never map into $A$.

6.2. The Cremer point case. We prove the Building Block Theorem 4.3 in the case of a quadratic polynomial $P$ with an invariant Cremer point $z_{0}$ of eigenvalue $e^{2 \pi i \alpha}$ such that the Julia set $J=J(P)=K(P)$ is a decomposable continuum. Since the Cremer point must be in $\overline{\mathcal{O}(c)}$, the only critical point $c$ is in $J$. Hence, $J$ must be a continuum. Since the Cremer point attracts the critical point, $J$ has no bounded complementary domains, so is a tree-like continuum. We take up the cases of (1) polynomials of degree $d>2$, and (2) periodic Cremer points, in a subsequent paper.

The first step in the proof of the Building Block Theorem for the Siegel disk case was to establish the existence of a Cantor set $A \subset \mathbb{T}$ generated by an irrational external angle $\theta$ such that $\operatorname{Im}\left(R_{\theta}\right)$ contained the critical value $v$. We later showed that $A$ had rotation number $\alpha$ under $\sigma$, where $\alpha$ was the rotation number of the Siegel disk, in Theorem 6.1. In the Cremer case, we begin with the following theorem, finding $\theta$ such that $\operatorname{Im}\left(R_{\theta}\right) \ni v$ and relating $\theta$ to $\alpha$. Our proof is inspired by the argument of Perez-Marco for Proposition II.11 in [24]; however, we have modified the argument.

Theorem 6.3. Let $P$ be a quadratic polynomial with Cremer fixed point $z_{0}$ with eigenvalue $e^{2 \pi i \alpha}$. Then there exists a $\theta \in \mathbb{T}$ such that $\operatorname{Im}\left(R_{\frac{\theta}{2}}\right)$ and $\operatorname{Im}\left(R_{\frac{\theta+1}{2}}\right)$ both 
contain $z_{0}$, its other inverse $z_{0}^{\prime}$, and the critical point c. Moreover, $\theta$ is irrational with rotation number $\alpha$ under $\sigma$.

Proof. By Yoccoz's Theorem (see Proposition II.12 in [24]), if $p_{n} / q_{n}$ are the convergents to $\alpha$ in its continued fraction expansion, then there exists a subsequence of convergents $\left(p_{n_{k}} / q_{n_{k}}\right)_{k \geq 0}$ such that $P$ has a periodic orbit $C_{k}$ of period $q_{n_{k}}$ of rotation number $p_{n_{k}} / q_{n_{k}}$ such that $C_{k}$ converges to $z_{0}$.

By Theorem 3.16, for any rational number $p / q$ there exist rational numbers $0<\theta^{-}(p / q)<\theta^{+}(p / q)<1$ generating a finite orbit of period $q$ under $\sigma$ which has rotation number $p / q$. Moreover,

$$
\begin{aligned}
& \left\{\theta^{-}(p / q), \theta^{+}(p / q), \frac{\theta^{+}(p / q)}{2}, \frac{\theta^{-}(p / q)+1}{2}\right\} \\
& \quad \subset \mathcal{O}_{\sigma}\left(\theta^{+}(p / q)\right) \subset\left[\frac{\theta^{+}(p / q)}{2}, \theta^{+}(p / q), \frac{\theta^{-}(p / q)+1}{2}\right]
\end{aligned}
$$

and

$$
\theta^{+}(p / q)-\theta^{-}(p / q)=\frac{1}{2^{q}-1}
$$

To simplify notation, let $\theta_{k}^{ \pm}=\theta^{ \pm}\left(p_{n_{k}} / q_{n_{k}}\right)$. Since $p_{n_{k}} / q_{n_{k}} \rightarrow \alpha$, it follows that $\theta_{k}^{ \pm} \rightarrow \theta=\theta(\alpha)$, where $\theta(\alpha)$ is the unique irrational corresponding to $\alpha$ given by the devil's staircase function, Figure 2 in Section 3.5. Since $P$ is quadratic, it is clear that the periodic points in $C_{k}$ are repelling, and hence, the external ray $R_{\theta_{k}^{+}}$lands on a periodic point that generates the orbit $C_{k}$, while $P^{i}\left(R_{\theta_{k}^{+}}\right)=R_{2^{i} \theta_{k}^{+}}$lands on the corresponding point of the orbit $C_{k}$, for $0 \leq i \leq q_{n_{k}}-1$. Hence, the invariant finite set $\mathcal{O}_{\sigma}\left(\theta_{k}^{+}\right)=\left\{\sigma^{i}\left(\theta_{k}^{+}\right) \mid 0 \leq i \leq q_{n_{k}}-1\right\}$ is contained in the interval $\left[\frac{\theta_{k}^{+}}{2}, \theta_{k}^{+}, \frac{\theta_{k}^{-}+1}{2}\right]$ and has rotation number $p_{n_{k}} / q_{n_{k}}$ under $\sigma$. By (25) above, let $z_{k} \in C_{k}$ be the point on which the external ray $R_{\theta_{k}^{+} / 2}$ lands. Let $C_{k}^{\prime}=P^{-1}\left(C_{k}\right) \backslash C_{k}$. Then $C_{k}^{\prime}$ converges to $z_{0}^{\prime}$. Moreover, the external ray $R_{\theta_{k}^{-} / 2}$ lands on a point $z_{k}^{\prime}$ of $C_{k}^{\prime}$.

It follows from equation (26) that

$$
\frac{\theta_{k}^{+}}{2}-\frac{\theta_{k}^{-}}{2}=\frac{1}{2} \frac{1}{2^{q_{k}}-1} .
$$

This implies that

$$
\lim \frac{\theta_{k}^{+}}{2}=\lim \frac{\theta_{k}^{-}}{2}=\frac{\theta}{2}
$$

We claim that

$$
z_{0}, z_{0}^{\prime} \in \operatorname{Im}\left(R_{\frac{\theta}{2}}\right) .
$$

To see this, let $\phi: \mathbb{C}_{\infty} \backslash \overline{\mathbb{D}} \rightarrow \mathbb{C}_{\infty} \backslash J$ be the Bötkher uniformization. (Recall that $J=J(P)=K(P)$.) Referring to the discussion of crosscuts and impressions in Section 3.1, let $\left\{Q_{k}\right\}_{k \geq 0}$ be a sequence of crosscuts of $U_{\infty}=\mathbb{C}_{\infty} \backslash J$ whose preimage under $\phi$ is a sequence of cross-cuts of $\mathbb{D}_{\infty}=\mathbb{C}_{\infty} \backslash \overline{\mathbb{D}}$ with the opposite endpoints of $\phi^{-1}\left(Q_{k}\right)$ being $\frac{\theta_{k}^{+}}{2}$ and $\frac{\theta_{k}^{-}}{2}$. Hence, the endpoints of the crosscut $Q_{k}$ are the landing points $z_{k}$ and $z_{k}^{\prime}$ of the rays $R_{\theta_{k}^{+} / 2}$ and $R_{\theta_{k}^{-} / 2}$, respectively. By (27), the sequence $\left\{\psi^{-1}\left(Q_{k}\right)\right\}_{k \geq 0}$ can be taken to converge to $\frac{\theta}{2}$. See Figure 4 . It follows from Lemma 3.1 that $\lim \sup \operatorname{Sh}\left(Q_{k}\right) \subset \operatorname{Im}\left(R_{\frac{\theta}{2}}\right)$. Since $z_{k}, z_{k}^{\prime} \in \operatorname{Sh}\left(Q_{k}\right), z_{k} \rightarrow z_{0}$, and $z_{k}^{\prime} \rightarrow z_{0}^{\prime}$, it follows that $z_{0}, z_{0}^{\prime} \in \lim \sup \operatorname{Sh}\left(Q_{k}\right)$, establishing claim (28). 


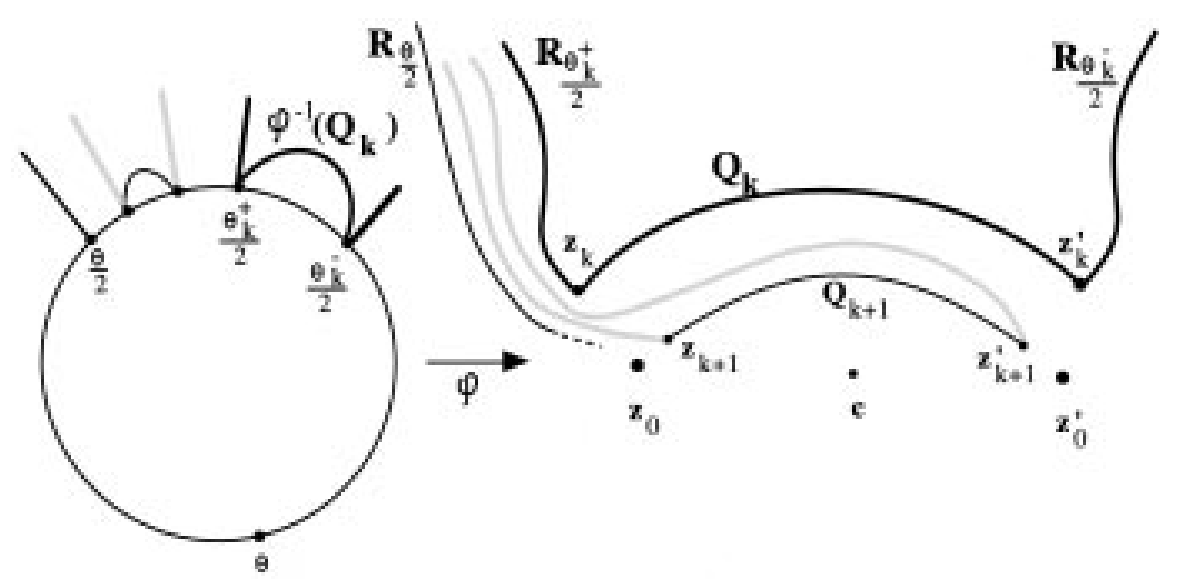

Figure 4. Critical impression in Cremer case

It is convenient to assume we have used the particular parameterization of quadratic polynomials of the form $P(z)=z^{2}+a$. Then the Cremer point and its preimage are $z_{0}$ and $-z_{0}$, and the critical point is $c=0$. It is easy to show that $J$ is symmetric about 0 as a center; that is, rotation of the plane about 0 by half a revolution carries $J$ onto $J, R_{t}$ onto $R_{t+\frac{1}{2}}$, and interchanges $z_{0}$ and $-z_{0}$. Hence, we also have

$$
z_{0},-z_{0} \in \operatorname{Im}\left(R_{\frac{\theta+1}{2}}\right)
$$

We claim that

$$
c \in \operatorname{Im}\left(R_{\frac{\theta}{2}}\right) \cap \operatorname{Im}\left(R_{\frac{\theta+1}{2}}\right) .
$$

To see that claim (30) holds, recall that $c=0 \in J$, and let $V$ be an open disk centered on 0 such that $\bar{V}$ misses both $z_{0}$ and $-z_{0}$. Let $y \in J \cap V$ be a repelling periodic point. Then some external ray $R_{s}$ lands on $y$. By symmetry, $R_{s+\frac{1}{2}}$ lands on $-y \in V$. Then $R_{s} \cup \bar{V} \cup R_{s+\frac{1}{2}}$ separates $\mathbb{C}$ between $z_{0}$ and $-z_{0}$. Since $\operatorname{Im}\left(R_{\frac{\theta}{2}}\right)$ is a continuum containing both $z_{0}$ and $-z_{0}$, it follows that $\operatorname{Im}\left(R_{\frac{\theta}{2}}\right) \cap V \neq \emptyset$. Since we can make $V$ arbitrarily small, $0 \in \operatorname{Im}\left(R_{\frac{\theta}{2}}\right)$. Similarly, $0 \in \operatorname{Im}\left(R_{\frac{\theta+1}{2}}\right)$.

6.2.1. Proof of Building Block Theorem 4.3. Let $P$ be a quadratic polynomial with Cremer fixed point $z_{0}$ with eigenvalue $e^{2 \pi i \alpha}$. By Theorem 6.3, there is an irrational $\theta \in \mathbb{T}$ such that the critical value $v \in \operatorname{Im}\left(R_{\theta}\right)$ and the rotation number of $\theta$ is $\alpha$. Since $J$ is a decomposable continuum, by Theorem 3.6 we know that $\operatorname{Im}\left(R_{\theta}\right)$ is a nowhere dense subcontinuum of $J$. Since $J$ is tree-like, so is each subcontinuum of $J$. We now proceed just as in the proof of the Siegel Building Block Theorem in Section 6.1. Let $U_{0}=\mathbb{C} \backslash \widehat{R}_{\theta}$. By claim (30), $c \in \operatorname{Im}\left(R_{\frac{\theta}{2}}\right) \cap \operatorname{Im}\left(R_{\frac{\theta+1}{2}}\right)$, so it follows that

$$
\mathbb{C} \backslash P^{-1}\left(\widehat{R}_{\theta}\right)=\mathbb{C} \backslash\left(\widehat{R}_{\frac{\theta}{2}} \cup \widehat{R}_{\frac{\theta+1}{2}}\right)
$$

has exactly two components, both unbounded, each of which maps conformally isomorphically onto $U_{0}$ under $P$. Let $U_{1}^{\prime}$ be the component of $\mathbb{C} \backslash\left(\widehat{R}_{\frac{\theta}{2}} \cup \widehat{R}_{\frac{\theta+1}{2}}\right)$ that contains $R_{\theta}$, and $U_{1}$ the other component (which necessarily contains $R_{0}$ ). 
We define the four collections $I_{n}, A_{n}, U_{n}$, and $B_{n}$, for $n \geq 0$, of subsets of $\mathbb{T}$ and $\mathbb{C}$, inductively, and then define $A=\bigcap_{n=0}^{\infty} A_{n}, \widehat{B}=\bigcap_{n=0}^{\infty} B_{n}$, and $B=$ $\widehat{B} \cap J=\bigcap_{n=0}^{\infty}\left(B_{n} \cap J\right)$ exactly as in Section 6.1. We shall show that $A$ and $B$ satisfy conclusions (1)-(10) of the theorem.

By Theorem 6.3 and Corollary 3.17, c, $z_{0}, z_{0}^{\prime} \in \operatorname{Im}\left(R_{\frac{\theta}{2}}\right) \cap \operatorname{Im}\left(R_{\frac{\theta+1}{2}}\right)$, and $A=\overline{\mathcal{O}(\theta)}$ is a Cantor set contained in the interval $\left[\frac{\theta}{2}, \theta, \frac{\theta+1}{2}\right] \subset \mathbb{T}$, having rotation number $\alpha$, and containing $\theta, \frac{\theta}{2}$, and $\frac{\theta+1}{2}$. Since the orbit of $\theta$ never enters any $I_{n}, n \geq 0$, the orbit of $R_{\theta}$ never enters any $U_{n}$. By the above, we have conclusions (5)-(8) of the theorem.

To establish the properties of the building block $B$ and the relationship of $B$ to $A$ we proceed for the most part just as in Section 6.1. The proof of conclusions (1)(4), (9), and (10) is based upon a series of claims parallel to, and worded exactly like, (15)-(24) with the following addition:

$$
\operatorname{Im}\left(R_{\frac{\theta}{2}}\right) \cup \operatorname{Im}\left(R_{\frac{\theta+1}{2}}\right) \subset B
$$

Assuming the parallel claims hold, the proof of conclusions (1), (2), the first part of (9), and (10) follows as in Section 6.1. Conclusions (3) and (4) follow from claim (31) and conclusion (2), since $c, z_{0}, z_{0}^{\prime} \in \operatorname{Im}\left(R_{\frac{\theta}{2}}\right)$. If $t \in A$ then there is a subsequence $\left\{\theta_{n}\right\}_{n=1}^{\infty}$ of $\mathcal{O}\left(\frac{\theta}{2}\right)$ which converges to $t$. By claim (31) and Lemma 1.2, since $z_{0}$ is fixed, $z_{0} \in \operatorname{Im}\left(R_{\theta_{n}}\right)$ for every $n$. By Lemma 1.1, $z_{0} \in R_{t}$, establishing conclusion (9).

The claims parallel to (15)-(24) are proved just as in Section 6.1. To see claim (31), note that by definition of $B_{1}, \operatorname{Im}\left(R_{\frac{\theta}{2}}\right) \cup \operatorname{Im}\left(R_{\frac{\theta+1}{2}}\right) \subset B_{1}$. Since $R_{\theta}$ never enters $U_{1}, R_{\frac{\theta}{2}}$ and $R_{\frac{\theta+1}{2}}$ never enter any component $W_{n}^{2}$ of $U_{n}$ for $n>1$. Hence, by Lemma 5.7, $\operatorname{Im}\left(R_{\frac{\theta}{2}}\right) \cup \stackrel{2}{\operatorname{Im}}\left(R_{\frac{\theta+1}{2}}\right) \subset \mathbb{C} \backslash W_{n}$. By induction on $n$, and intersecting over the finitely many complements of components of $U_{n}$, we get $\operatorname{Im}\left(R_{\frac{\theta}{2}}\right) \cup \operatorname{Im}\left(R_{\frac{\theta+1}{2}}\right) \subset$ $B_{n}$. Claim (31) follows by intersecting over $n$ and with $J$.

6.2.2. Proof of Skeleton Corollary 4.4. The proof that the Cremer building block $B$ generates a skeleton $S$ for the corresponding Julia set is the same as in Section 6.1.2.

\section{Concluding Questions}

7.1. Siegel disk case. Let $P$ be a quadratic polynomial with Siegel disk $\Delta$. Let $B$ denote the Siegel building block, $S=\bigcup_{n=0}^{\infty} P^{-n}(B)$ the skeleton, and $C$ the critical continuum joining copies of $B$ in $S$.

1. Is the orbit of the critical point $c$ dense in $B$ ?

2. Does any external ray land on $B$ or on $\partial \Delta$ ?

3. Do the copies of $B$ (and of $C$ ) in $S$ have diameter going to 0 as $n$ goes to $\infty$ ?

4. Are the components of $J \backslash S$ points? If so, what is the dimension of $J \backslash S$ ?

5 . Does $J$ contain points of local connectivity?

6. To what kind(s) of prime end does $t \in A$ correspond?

7. Suppose $t$ is a point in $\mathbb{T}$ whose forward orbit never enters the Cantor set $A$. What kind of prime end corresponds to $t$ ?

8. If $s \neq t \in \mathbb{T}$, and neither ever maps into $A$, then is $\operatorname{Im}\left(R_{s}\right) \cap \operatorname{Im}\left(R_{t}\right)=\emptyset$ ?

9. Can $B$ contain a copy of a hairy circle? (A hairy circle is a circle with uncountable many hairs of all lengths $\leq 1$ attached at a dense set; see [1] for an exact definition) 
7.2. Cremer case. Let $P$ be a quadratic polynomial with Cremer point $z_{0}$. One may raise the same questions in the Cremer case regarding $B, S$, and $C$ as for the Siegel disk case. In addition, the following questions arise in the Cremer case:

10. Does the orbit of the critical point $c$ accumulate on the preimage $z_{0}^{\prime}$ of the Cremer point?

11. Does any external ray land on $z_{0}$ ?

12. Is $z_{0}$ the impression of a prime end of the first kind?

13. Is there any external angle whose impression contains $B$ ?

14. Is the impression of each external angle in $A$ nowhere dense in $B$ ?

15. Do the impressions in $B$ have only $z_{0}$ in common?

16. Can $B$ contain a copy of a Cantor bouquet? (A Cantor bouquet is a smooth fan with a dense set of endpoints, see [6]. Similar objects were first introduced in [11].)

We would like to thank Jan Kiwi for helpful comments about an earlier version of this paper.

\section{REFERENCES}

[1] J. M. Aarts and L. G. Oversteegen. The geometry of Julia sets Trans. A. M. S., 338:897-918, 1993. MR 93j:30021

[2] A. F. Beardon. Iteration of rational functions, volume 132 of Graduate texts in mathematics. Springer-Verlag, 1991. MR 92j:30026

[3] A. D. Brjuno. Convergence of transformations of differential equations to normal forms. Dokl. Aked. Nauk. USSR, 165:987-989, 1965; English transl., Soviet Math. Dokl. 6:1536-1538, 1965. MR 33:325

[4] A. D. Brjuno. Analytical form of differential equations. Trans. Moscow Math. Soc., 25:131288, 1971. MR 51:13365

[5] A. D. Brjuno. Analytical form of differential equations. Trans. Moscow Math. Soc., 26:199239, 1972. MR 51:13365

[6] W. T. Bula and L. G. Oversteegen. A characterization of smooth Cantor bouquets. Proc. A. M. S., 108:529-534, 1990. MR 90d:54066

[7] S. Bullett and P. Sentenac. Ordered orbits of the shift, square roots, and the devil's staircase. Math. Proc. Camb. Phil. Soc., 115:451-481, 1994. MR 95j:58043

[8] L. Carleson and T. W. Gamelin. Complex Dynamics. Universitext: Tracts in Mathematics. Springer-Verlag, 1993. MR 94h:30033

[9] H. Cremer. Zum zentrumproblem. Math. Ann., 98:151-163, 1928.

[10] R. L. Devaney and L. R. Goldberg. Uniformization of attracting basins. Duke Math. J., 55:253-266, 1987. MR 88h:30035

[11] R. L. Devaney and M. Krych. Dynamics of exp(z). Ergodic Theory and Dyn. Sys., 4:35-52, 1984. MR 86b:58069

[12] A. Douady. Systemes dynamiques holomorphes. Sém. Bourbaki, exp. 599, Astérisque, 105106:39-63, 1983. MR 85h:58090

[13] A. Douady. Disques de Siegel et anneaux de Herman. Sém. Bourbaki, exp. 677, Astérisque, 152-153(4):151-172, 1988. MR 89g:30049

[14] E. Dyer. Irreducibility of the sum of the elements of a continuous collection of continua. Duke Math. J., 20: 589-592, 1953. MR 15:335f

[15] L. Goldberg and J. Milnor. Fixed points of polynomial maps, Part II, Fixed point portraits. Ann.Sci.Ec.Norm.Sup. 4 serie 26, 26:51-98, 1993. MR 95d:58107

[16] M. R. Herman. Recent results and some open questions on Siegel's linearization theorem of germs of complex analytic diffeomorphisms of $\mathbb{C}^{n}$ over a fixed point. In Proc. of the VIII Int. Cong. Math. Phys., volume 34, pages 138-198, Singapore, 1986. World Scientific. MR 89k:32013

[17] J. Kiwi. Non-accessible critical points of Cremer polynomials. Preprint.

[18] K. Kuratowski. Topology II. Academic Press, New York, 1968. MR 41:4467 
[19] J. C. Mayer and J. T. Rogers, Jr. Indecomposable continua and the Julia sets of polynomials. Proc. A. M. S., 117:795-802, 1993. MR 93d:58138

[20] J. Milnor. Dynamics in one complex variable: introductory lectures. Technical Report 5, SUNY-Stony Brook, 1990. Institute for Mathematical Sciences.

[21] J. Milnor. Locally connected Julia sets: Expository lectures. Technical Report 11, SUNYStony Brook, 1992. Institute for Mathematical Sciences.

[22] R. Perez-Marco. Sur les dynamiques holomorphes non linearisables et une conjecture de V.I. Arnold. Ann.Scient. Ec. Norm. Sup. 4 serie, 26:565-644, 1993. MR 85a:58103

[23] R. Perez-Marco. Fixed points and circle maps. Acta Math. 179:243-294, 1997. CMP 98:08

[24] R. Perez-Marco. Topology of Julia sets and hedgehogs. Technical Report 48, Universite de Paris-Sud, 1994.

[25] C. L. Petersen. Local connectivity of some Julia sets containing a circle with an irrational rotation. IHES/M/94/26, 1994. Acta Math. 177:163-224, 1996. CMP 97:10

[26] J. T. Rogers, Jr. Singularities in the boundaries of local Siegel disks. Ergodic Theory and Dynamical Systems, 12:803-821, 1992. MR 93m:58061

[27] J. T. Rogers, Jr. Critical points on the boundaries of Siegel disks of polynomials. Bull. A. M. S., 32:317-321, 1995. MR 96a:30032

[28] J. T. Rogers, Jr. Diophantine conditions imply critical points on the boundaries of Siegel disks of polynomials. Comm. Math Phys., To appear.

[29] N. E. Rutt. Prime ends and indecomposability. Bull. A. M. S., 41:265-273, 1935.

[30] C. L. Siegel. Iteration of analytic functions. Ann. of Math., 43:607-612, 1942. MR 4:76c

[31] D. Sørensen. Local connectivity of quadratic Julia sets. Master's thesis, Mathematical Institute, The Technical University of Denmark, 1992.

[32] D. Sørensen. Complex dynamical systems. PhD thesis, Mathematical Institute, The Technical University of Denmark, 1994.

[33] D. Sullivan. Conformal Dynamical Systems, volume 1007 of Springer Lecture Notes, pages 725-752. Springer Verlag, NY, 1983. MR 85m:58112

[34] D. Sullivan. Quasiconformal homeomorphisms and dynamics I, solution of the Fatou-Julia problem on wandering domains. Annals of Math., 122:401-418, 1985. MR 87i:58103

[35] J. C. Yoccoz. Linearisation des germes de difféomorphismes holomorphes de (C, 0). C.R. Acad. Sci. Paris, 306:55-58, 1987. MR 89i:58123

[36] J. C. Yoccoz. Théorème de Siegel, nombres de Bruno et polynomes quadratiques. Petits diviseurs en dimension 1, Asterisque, 231:3-88, 1995. MR 96m:58214

Technical University of Crete, Chania, Greece

E-mail address: mgrysp@euclid.aml.tuc.gr

University of Alabama at Birmingham, Birmingham, Alabama 35294-1170

E-mail address: mayer@math.uab.edu

E-mail address: overstee@math.uab.edu 